\title{
Discovery and Development of a Novel mPGES-I/ 5-LOX Dual Inhibitor LFA-9 for Prevention and Treatment of Chronic Inflammatory Diseases
}

This article was published in the following Dove Press journal: Journal of Inflammation Research

\author{
Nagendra Sastri Yarla' \\ Gopal Pathuri, ${ }^{1,2}$ \\ Hariprasad Gali ${ }^{2}$ \\ Simon Terzyan $\left(\mathbb{D}^{3}\right.$ \\ Janani Panneerselvam' \\ Parthasarathy Chandrakesan ${ }^{4}$ \\ Marcus Tullius Scotti ${ }^{5}$ \\ Courtney Houchen ${ }^{4}$ \\ Venkateshwar Madka' \\ Chinthalapally $\vee \mathrm{Rao}{ }^{1,6}$ \\ 'Center for Cancer Prevention and Drug \\ Development, Hem-Onc Section, \\ Department of Medicine, Stephenson \\ Cancer Center, University of Oklahoma \\ Health Sciences Center, Oklahoma City, \\ OK 73104, USA; ${ }^{2}$ College of Pharmacy, \\ University of Oklahoma Health Sciences \\ Center, Oklahoma City, OK 73I04, USA; \\ ${ }^{3}$ Laboratory of Biomolecular Structure \\ and Function; Department of \\ Biochemistry and Molecular Biology, The \\ University of Oklahoma Health Sciences \\ Center, Oklahoma City, OK 73I04, USA; \\ ${ }^{4}$ Division of Digestive Diseases and \\ Nutrition, Department of Medicine, \\ University of Oklahoma Health Sciences \\ Center, Oklahoma City, OK 73I04, USA; \\ ${ }^{5}$ Laboratory of Cheminformatics, \\ Program of Natural and Synthetic \\ Bioactive Products (PgPNSB), Health \\ Sciences Center, Federal University of \\ Paraíba, João Pessoa, PB, Brazil; ${ }^{6}$ VA \\ Medical Center, Oklahoma City, OK \\ 73I04, USA
}

Correspondence: Chinthalapally V Rao Tel + I 405-27I-3224

Email CV-Rao@ouhsc.edu
Background: Non-steroidal anti-inflammatory drugs, cyclooxygenase (COX)-2 selective inhibitors, have been explored for prevention and treatment of several inflammatory chronic conditions including arthritis, and cancer. However, the long-term use of these drugs is associated with gastrointestinal, renal, and cardiovascular side effects. Later, COX/5-lipoxygenase (5-LOX) dual inhibitors (eg, licofelone) have been developed but did not enter into the market from the clinical trails due to COX-1/2 inhibition-associated side effects. Hence, targeting microsomal prostaglandin E synthase-1 (mPGES-1) and 5-LOX can be an ideal approach while sparing COX-1/2 activities for development of the next generation of anti-inflammatory drugs with better efficacy and safety.

Materials and Methods: In silico (molecular modelling) studies were used to design a mPGES1/5-LOX dual inhibitory and COX-1/2 sparing lead molecule licofelone analogue-9 (LFA-9) by modifying the pharmacophore of licofelone. In vitro cell-free enzymatic (mPGES-1, 5-LOX, COX$1 / 2$ ) assays using fluorometric/colorimetric methods and cell-based assays (LPS-induced PGE $_{2}$, $\mathrm{LTB}_{4}$, and $\mathrm{PGI}_{2}$ productions from macrophages) using ELISA technique, isothermal calorimetry, and circular dichroism techniques were performed to determine the mPGES-1/5-LOX inhibitory efficacy and selectivity. Anti-inflammatory efficacy of LFA-9 was evaluated using a carrageenan (inflammogen)-induced rat paw edema model. Infiltration/expression of CD68 immune cells and TNF- $\alpha$ in paw tissues were evaluated using confocal microscope and immunoblot analysis. Anticancer effect of LFA-9 was evaluated using colon spheroids in vitro.

Results: LFA-9 inhibited mPGES-1/5-LOX and their products $\mathrm{PGE}_{2}$ and $\mathrm{LTB}_{4}$, spared COX-1/2 and its product PGI $_{2}$. LFA-9 bound strongly with human mPGES-1/5-LOX enzymes and induced changes in their secondary structure, thereby inhibited their enzymatic activities. LFA-9 inhibited carrageenan-induced inflammation $(70.4 \%)$ in rats and suppressed CD68 immune cell infiltration $(P \leq 0.0001)$ and TNF- $\alpha$ expression. LFA-9 suppressed colon tumor stemness $(60.2 \%)$ in vitro through inhibition of $\mathrm{PGE}_{2}(82 \%)$ levels.

Conclusion: Overall study results suggest that LFA-9 is a mPGES-1/5-LOX dual inhibitor and showed anti-inflammatory and colorectal cancer preventive activities, and warranted detailed studies.

Keywords: mPGES-1/5-LOX dual inhibitor, LFA-9, drug design, anti-inflammatory agent, cancer chemoprevention

\section{Introduction}

Non-steroidal anti-inflammatory drugs (NSAIDs) such as aspirin, naproxen, and sulindac, cyclooxygenase (COX)-2 selective inhibitors (eg, celecoxib), have been explored for prevention and treatment of several chronic inflammatory conditions including arthritis, and cancer. However, the application of these drugs, particularly 
long-term use, is associated with gastrointestinal, renal, and cardiovascular side effects and metabolic pathway diversion of substrate arachidonic acid into pro-inflammatory leukotrienes by activation of 5-LOX enzyme. ${ }^{1,2}$ Later, COX and 5-lipoxygenase (CLOXIB) dual inhibitors (eg, licofelone) were developed but most of them did not enter into the market from clinical trails due to safety concerns. ${ }^{3}$ These side effects are due to COX-1/2 inhibition and their downstream product prostacyclin $\mathrm{I}_{2}\left(\mathrm{PGI}_{2}\right)$ and renal function disruption. ${ }^{3,4}$ Hence, mechanistic studies suggest that sparing COX-1/2 and selectively targeting microsomal prostaglandin E synthase-1 (mPGES-1) and 5-LOX would be a safer and effective strategy to inhibit $\mathrm{PGE}_{2}$ and leukotrienes (LTs), respectively, and to spare $\mathrm{PGI}_{2}$. In this scenario, several mPGES-1/5-LOX dual inhibitors have been developed for the next generation of antiinflammatory drugs (Figure 1A)..$^{5-7}$

Licofelone (formerly know as ML-3000), a potent inhibitor of COX-1/2, 5-LOX, and weak inhibitor of mPGES-1 inhibitor, has been evaluated in clinical trials for several inflammatory and oncologic diseases ${ }^{8,9}$ (Figure 1). Previously, we have shown that licofelone had highly preventive efficacious effects in several preclinical models of colon, lung, pancreatic and urinary bladder cancer. ${ }^{10-13}$ However, its COX-inhibition-associated side effects have somewhat limited its clinical applications. ${ }^{14,15}$ Licofelone is a potential pharmacophore with which to develop mPGES-1/5-LOX dual inhibitory, and COX-1/2 activity sparing analogs. ${ }^{8}$ Previously, several groups made efforts to develop licofelone analogs by modifying its pharmacophore at the carboxylic end to yield mPGES-1/5-LOX inhibitor with COX-1/2 sparing property, but were not fully successful in developing dual mPGES-1/5-LOX inhibitors for clinical translation. ${ }^{8}$ In addition, the structural variability and the absence of crystal structures of the target enzymes, among experimental animals (rodents) and humans are limiting the lead optimization and preclinical development and clinical translation of mPGES-1/ 5-LOX inhibitory agents, ${ }^{16,17}$ (Supplemental Information Figures 1-4; Supplemental Information Table 1). So far, none of the reported potent inhibitors of human/mouse/rat specific mPGES-1 and 5-LOX inhibitors for pre-clinical evaluation and clinical translation. ${ }^{18}$

Thus, the main aim of the present work was to modify the licofelone pharmacophore at its carboxyl end using a hit library (active fragments over 1000) through fragmentbased drug design strategy to target mPGES-1/5-LOX while sparing COX-1/2 activities of human, mouse, and rat to facilitate its preclinical development and further to clinical translation. In this study, we modeled the three-dimensional structures of some of the target proteins as the crystal structures of some target proteins are not available. Among over 1,000 designed molecules, an optimized lead molecule (licofelone analogue (LFA-9) (Figure 1B) was selected and identified as a potential candidate for development of mPGES-1/ 5-LOX inhibitor based on molecular docking studies and druggable properties. Further, LFA-9 was synthesized and in vitro cell-free and cell-based assays were performed to determine its selective inhibitory effect on human/mouse/rat mPGES-1/5-LOX and their respective products $\mathrm{PGE}_{2}$ and $\mathrm{LTB}_{4}$, and sparing effect on COX-1/2 activity and $\mathrm{PGI}_{2}$ production. Isothermal calorimetric (ITC) and circular dichroism (CD)-based studies were performed to determine the binding affinity and structural changes induced by LFA- 9 on human mPGES-1/5-LOX enzymes. Further, in vivo antiinflammatory activity of LFA- 9 was evaluated using carrageenan (inflammogen)-induced rat paw edema model. In addition, the anti-cancer effect of LFA- 9 was evaluated by in vitro assay using colon spheroids, since inflammation causes the tumorigenesis of colorectal cancer. ${ }^{19,20}$

\section{Materials and Methods}

General Information

Human mPGES-1 was expressed and purified from Escherichia coli Rosetta $^{21}$ and also purchased from CloudClone Corp. (Item No. RPA167Hu01) (Houston, TX, USA). ELISA kits for $\mathrm{PGE}_{2}$ (Item No. 500141), $\mathrm{LTB}_{4}$ (Item No. 520111), $\mathrm{PGI}_{2}$ ( $\alpha$-keto prostaglandin) (Item No. 515211), and $\mathrm{TXB}_{2}$ (Item No. 501020) quantification, recombinant human COX-1 (Item No. 17616) and $\mathrm{PGH}_{2}$ (Item No. 17020) were purchased from Cayman Chemical (Ann Arbor, MI, USA). Glutathione (GSH) (Cat No. GI317), lamda-carrageenan (Cat. No. sc-216084) was purchased from Santa Cruz Biotechnology (Dallas, TX, USA). Fluorometric assay kits for 5-LOX (cat no. K980), COX-2 (Cat No. K547), and COX-1 (Cat No. K548) activity assays were purchased from Biovision, Inc. (Milpitas, CA, USA) and Cloud-Clone Corp. (Houston, TX, USA). CD68 antibody with Alexa Fluor $^{\circledR} 700$ ] (Novus Biologicals, CA, USA; 600985), CD68 antibody (catalog sc-9139; Santa Cruz, Dallas, TX, USA), TNF- $\alpha$ antibody (Bioss, Woburn, MA, USA), (BS-2018R), beta-actin (abcam, ab8227) are purchased from respective vendors and used in the study. Mouse- and rat-specific COX, mPGES-1 enzymes were isolated from their vas deferens and seminal vesicles. ${ }^{22-24}$ Licofelone and LFA-9 were 


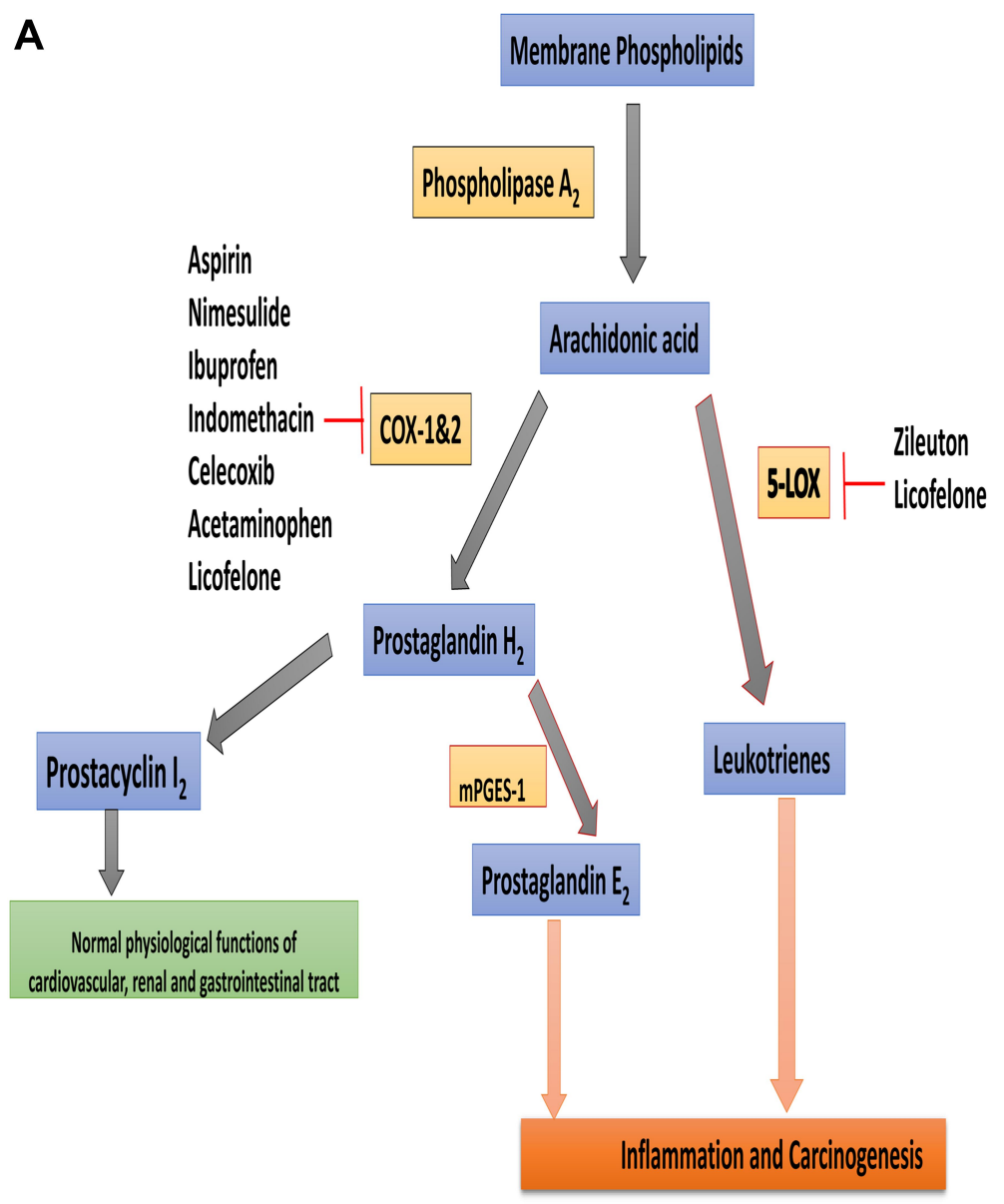

B
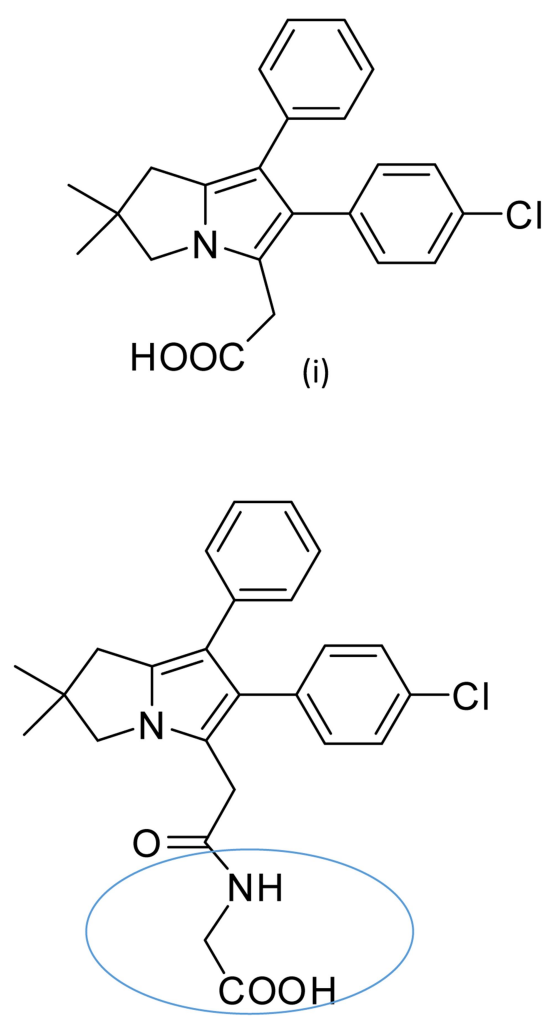

(ii)

Figure I (A) Arachidonic acid pathway and its inhibitors in clinical use for inflammatory and oncological diseases. (B) Chemical structures of licofelone (i) and LFA-9 (ii).

synthesized in the laboratory and characterized using advanced spectroscopic techniques. ${ }^{25}$

Reversed-phase HPLC analyses were performed on a Shimadzu HPLC equipped with a LC-10ADvp pump, SCL 10Avp system controller and SPD-M10Avp PDA detector. All the operations were controlled by Shimadzu EZ start 7.2.1 software in Windows XP system. HPLC solvents consisted of water containing $0.1 \%$ trifluoroacetic acid (solvent A) and acetonitrile containing $0.1 \%$ trifluoroacetic acid (solvent B). A Sonoma C18 (ES Industries, $10 \mu \mathrm{m}$, $100 \AA$, 4.6 x $250 \mathrm{~mm}$ ) column was used with a flow rate of $1 \mathrm{~mL} / \mathrm{min}$. The HPLC isocratic method solvent composition of $30 \% \mathrm{~A}$ and $70 \% \mathrm{~B}$ for 15 minutes. The PDA detector was set between 190 and $800 \mathrm{~nm}$ and analysis was done at $254 \mathrm{~nm}$.

${ }^{1} \mathrm{H}$ and ${ }^{13} \mathrm{C}$ NMR spectroscopy was performed in the University of Oklahoma College of Pharmacy NMR Facility using a Varian (Palo Alto, CA, USA) Mercury VX-300 NMR Spectrometer. Electrospray mass spectral analysis was performed by the University of Oklahoma
Health Sciences Center (OUHSC) Molecular BiologyProteomics Facility using a Waters Synapt G2-s mass spectrometer.

\section{Synthesis and Characterization of (2-[[2-} [2-(4-Chlorophenyl)-6,6-Dimethyl-IPhenyl-5,7-Dihydropyrrolizin-3-YI]acetyl] amino]acetic Acid) (LFA-9)

The synthesis of LFA-9 was achieved in a two-step synthetic strategy (Supplement Figure 5A) as per previously patented procedure. ${ }^{25}$ Briefly, to a solution of licofelone (1) $(28.45 \mathrm{~g}, 75 \mathrm{mmol})$ in dichloromethane $(300 \mathrm{~mL})$ was added $N$-hydroxysuccinimide $(9.50 \mathrm{~g}, 82.5 \mathrm{mmol})$ in dichloromethane $(300 \mathrm{~mL})$ and $N, N$ '-dicyclohexylcarbodiimide (17.03 g, $82.5 \mathrm{mmol})$ in dichloromethane (100 $\mathrm{mL}$ ). The reaction mixture was stirred at room temperature overnight. The progress of the reaction was monitored by thin layer chromatography (TLC). After completion of the reaction, $N, N^{\prime}$-dicyclohexylurea (byproduct) was filtered 
on a sintered funnel, and filtrate was evaporated on a rotary evaporator under reduced pressure to obtain licofelone-NHS in a quantitative yield (2). This compound was used immediately for the next step without further purification.

To a solution of licofelone-NHS (35.79 g, $75 \mathrm{mmol})$ in $N, N$-dimethylformamide $(650 \mathrm{~mL})$ was added glycine (5.63 g, $75 \mathrm{mmol})$ in $0.2 \mathrm{M} \mathrm{Na}_{2} \mathrm{HPO}_{4}(100 \mathrm{~mL}, \mathrm{pH} 8.0)$. The reaction mixture was stirred at room temperature overnight. The progress of the reaction was monitored by TLC. After completion of the reaction, solvents were evaporated on a rotary evaporator under reduced pressure, and the product was extracted into ethyl acetate $(2 \times 300$ $\mathrm{mL})$. The combined organic layer was washed with water $(2 \times 100 \mathrm{~mL})$ and with brine $(2 \times 100 \mathrm{~mL})$. The organic layer was dried over sodium sulfate, and solvents were evaporated on a rotary evaporator under reduced pressure to obtain the crude LFA-9 (3). The crude compound was purified on a silica gel column by eluting with ethyl acetate to obtain the pure LFA-9 as a white solid (16.5 g, $50.3 \%$ ). Alternatively, the crude compound was purified by recrystallization using an ethyl acetate/hexane solvent mixture (26.4 g, 80.5\%). Purity of LFA-9 as determined by high performance liquid chromatography (HPLC). Melting point, ${ }^{1} \mathrm{H}$ and ${ }^{13} \mathrm{C}$, NMR and high-resolution mass spectroscopic (HRMS) techniques were used to determine the chemical structure of LFA-9.

\section{Cell-Free Enzyme Assays \\ 5-LOX Assay}

5-LOX (human/mouse/rat) assays were performed using fluorometric-based 5-LOX inhibitor screening kits according to the manufacturer's instructions (Biovision, Inc., Milpitas, CA, USA). 5-LOX converts arachidonic acid into 5-hydroperoxyeicosatetraenoic acid (5-HPETE). This intermediate interacts with the specific probe and leads to fluorescence emission, which can be measured at $E x / E m$ $500 / 536 \mathrm{~nm}$.

5-LOX + (Arachidonic acid) Substrate $\rightarrow$ 5-HPETE (Intermediate) + Probe $\rightarrow$ Fluorescence (Ex/Em: 500/ $536 \mathrm{~nm})$

Different concentrations of LFA-9 or licofelone (0.1$100 \mu \mathrm{M})$ were incubated with 5-LOX (2 $\mu \mathrm{U}$ (units)) in enzymatic assay buffer mixture and the addition of substrate $(20 \mu \mathrm{L}$ of $100 \mu \mathrm{M}$ arachidonic acid). Then, we read fluorescence $(E x / E m 500 / 536 \mathrm{~nm})$ kinetically from 0-10 minutes. The untreated 5-LOX enzymatic wells were considered a control (100\% activity of the enzyme). We calculated relative fluorescence units $(\triangle R F U)$ for all samples by subtracting RFU at time $\mathrm{t} 1$ (initial) from RFU at time $\mathrm{t} 2$ (end) (10 minutes). Slopes for all samples were calculated by dividing $\Delta$ RFU by time $\Delta \mathrm{t}$ (t1-t2). $0.01 \%$ DMSO or methanol (solvents used to dissolve the compound) was used for solvent control. Non-enzymatic $\triangle \mathrm{RFU}$ were measured and subtracted from the control and test values. Experiments were conducted in triplicate. Percent inhibition of 5-LOX activity was calculated based on the following equation:

$\%$ inhibition $=($ slope of the enzyme control - slope of test compound/slope of enzyme control) x 100\% relative activity $=$ [slope of test/slope of enzyme control] $\mathrm{x} 100$

\section{mPGES-I Assay}

An mPGES-1 assay was performed according to the method described previously by Kim et al. ${ }^{21}$ The appropriate amount of mPGES-1 in $100 \mu \mathrm{L}$ of assay buffer (15 $\mathrm{mM}$ Tris- $\mathrm{HCl}, \mathrm{pH} 7.4$, and $1 \mathrm{mM} \mathrm{GSH}$ ) was added to each well of a 96-well plate. The plate was incubated for 15 minutes at $20^{\circ} \mathrm{C}$ and an enzymatic reaction was initiated by addition of $20 \mu \mathrm{L}$ of cold $\mathrm{PGH}_{2}$ (final conc. $2.8 \mu \mathrm{M}$ ). The plate was further incubated for 45 seconds at room temperature. Incubation was immediately terminated by adding $20 \mu \mathrm{L}$ of $\mathrm{SnCl}_{2}$ solution in $1 \mathrm{~N} \mathrm{HCl}$. $\mathrm{PGE}_{2}$ levels were estimated using $\mathrm{PGE}_{2}$-monoclonal antibody-based ELISA kits (Cayman Chemical, Ann Arbor, MI, USA). Enzyme inhibitory activity of control (without compound) and/or test chemical compounds was measured by the incubation of $2 \mu \mathrm{L}$ of a compound $(0.1-100 \mu \mathrm{M})$ in buffer with mPGES-1 for 20 minutes prior to the addition of substrate $\mathrm{PGH}_{2}$. Percent inhibition (triplicate) was calculated from $\mathrm{PGE}_{2}$ reduction in test wells (compound-treated) compared with control (untreated). ${ }^{21}$ The results were expressed in $\mathrm{IC}_{50}$.

\section{COX-I/2 Assay}

COX-1/2 (human/mouse/rat) assays were performed using a fluorometric-based COX inhibitor screening kit according to the manufacturer's instructions (Biovision, Inc., Milpitas, CA, USA). Rat and mouse COX-1/2 were isolated from seminal vesicles. The assays were performed using kits, which are provided with isoform-specific inhibitors. COX converts arachidonic acid into $\mathrm{PGG}_{2}$. This intermediate interacts with a specific probe that leads to fluorescence emission, which can measure fluorescence at Ex/Em 535/587 nm. 
Different concentrations of LFA-9 or licofelone (0.1$100 \mu \mathrm{M})$ were incubated with $\mathrm{COX}(2 \mu \mathrm{U})$ in enzymatic assay buffer mixture with addition of substrate $(20 \mu \mathrm{L}$ of $100 \mu \mathrm{M}$ arachidonic acid). Then, we read fluorescence (Ex/Em 535/587 nm) kinetically from 0-10 minutes. Untreated COX enzymatic mixture was considered a control (100\% activity of the enzyme). We calculated relative fluorescence units $(\triangle \mathrm{RFU})$ for all samples by subtracting RFU at time t1 from RFU at time t2 (10 minutes). Slopes for all samples were calculated by dividing $\triangle \mathrm{RFU}$ by time $\Delta \mathrm{t}$ (t1-t2). $0.01 \%$ DMSO or methanol (solvents used to dissolve the compound) was used for solvent control. Nonenzymatic $\triangle \mathrm{RFU}$ were measured and subtracted from the control and test values. Experiments were conducted in triplicate. Percent inhibition of COX-1/2 activity was calculated.

\section{Cell-Based Assay Using Human/Mouse/ Rat Macrophages}

Human macrophages were obtained from commercially derived THP1-human monocytes (ATCC; batch number:70005912) using phorbol 12-myristate 13 -acetate. ${ }^{26}$ Rat/mouse peritoneal macrophages were isolated as per the protocol described previously. ${ }^{27}$ Briefly, after euthanasia to the animals, the superficial skin was disinfected with $70 \%$ ethanol, and then the skin on the abdomen was removed while the peritoneum was left intact. Animals were then injected with $5 \mathrm{~mL}$ ice-cold Dulbecco's Modified Eagle Medium (DMEM) media, followed by gentle shaking to allow for the distribution of the lavage fluid throughout the peritoneal cavity. The DMEM lavage fluid was then collected back using a 5-mL syringe attached to a 25 -gauge needle. Peritoneal macrophages were determined by adherence to glass slides ( 1 hour, $37^{\circ} \mathrm{C}$ ). Unattached cells were washed off and attached cells were studied for morphology under light microscopy for the experiment. Cell number and viability was confirmed based on the Trypan-blue exclusion method. Then, cells were washed three times with DMEM $+10 \%$ fetal bovine serum and seeded in 96- or 24-well plates with an equal number (density of 10,000 cells/well for 96-well; 40,000 cells/well for 24-well) treated with non-toxic doses of LFA-9 and licofelone, and then stimulated with LPS (10 $\mu \mathrm{g} / \mathrm{mL}$ ) for 24 hours. The culture supernatant was collected for estimation of exogenous $\mathrm{PGE}_{2}, \mathrm{PGI}_{2}$, and $\mathrm{LTB}_{4}$ levels. Percent inhibition and $\mathrm{IC}_{50}$ were calculated.

\section{Molecular Modelling and Docking Studies}

Human mPGES-1 (trimeric form) (PDB ID: 4YL3), human 5-LOX (PDB ID: 3O8Y), human COX-2 (PDB ID: 5KIR), and mouse COX-2 (PDB ID: 4COX) three-dimensional structures were obtained from Protein Data Bank and used in docking studies. mPGES-1 (mouse and rat), 5-LOX (mouse and rat), and COX-1 (human, mouse and rat), COX-2 (mouse) crystal structures were not available. Thus, three-dimensional structures of these proteins were modelled for molecular docking studies. Crystal structures of the aforementioned proteins were modelled based on their protein sequences (source: NCBI protein) using SwissModel via the ExPASy web server (https://swissmodel. expasy.org/). We validated the structure through Ramachandran plots using Rampage (http://mordred.bioc. cam.ac.uk/ rapper/rampage.php). Sequence similarity between different species of mPGES-1 was determined using the NCBI blast program (https://blast.ncbi.nlm.nih gov/Blast.cgi). Co-crystallized ligands and water molecules were removed from target protein using ArgusLab. Ligands were prepared using ChemOffice (Cambridge, UK). Energy minimization was done using molecular mechanics, which was executed until the root mean square value reached below $0.001 \mathrm{Kcal} / \mathrm{mol}$. Such energy-minimized ligands and receptors were used for docking studies using GEMDOCK (Generic Evolutionary Method for molecular DOCKing), which is a generic evolutionary method with an empirical scoring function for molecular docking (protein-ligand docking). A population size of 300 with 70 generations and three solutions were used in docking accuracy setting. ${ }^{28}$ PyMol was used for better visualization of interactions. ${ }^{29}$

\section{Binding Assay Using Isothermal Titration Calorimetry}

Isothermal titration calorimetry (ITC) is a well-established technique that can determine all the thermodynamic parameters, including affinity, of a binding interaction between an enzyme and a small molecule. ${ }^{30}$ ITC (VP-ITC manufactured by MicroCal [now part of GE Healthcare]) was used to evaluate the binding efficacy of LFA-9 on the target enzymes. Compounds (up to $1 \mathrm{mM}$ ) and proteins $(1 \mathrm{mM})$ are dissolved in phosphate buffered saline and degassed before use in the experiment. A series of injections of the compound were administered to an enzymecontaining well. The heat signal will approach zero as the limiting reactant becomes saturated. Accurate fitting of the 
isotherm was performed using Origin software to calculate the binding affinity dissociation constant (KD). ${ }^{30}$

\section{Determination of Conformational}

\section{Changes in the Secondary Structure of} mPGES-I/5-LOX/COXs Using the

\section{Circular Dichroism (CD) Technique}

Native and LFA-9 $(0.1-10 \mu \mathrm{M})$-treated mPGES-1/5-LOX/ COXs were dissolved in phosphate-buffered saline ( $\mathrm{pH}$ 7.4) with a final protein concentration of $200 \mu \mathrm{M}$. The samples were transferred to a 1-mm path length quartz cuvette. Circular dichroism spectra in the wavelength range 185$300 \mathrm{~nm}$ was acquired in-house with a Jasco 715 Circular Dichroism (CD) Spectropolarimeter (Jasco Corp., Japan) using a bandwidth of $1 \mathrm{~nm}$ and a response time of 1 second. Multiple scans were accumulated for each sample. All spectra were corrected by subtraction of buffer blanks. The percent change in secondary structure of proteins was calculated using a CAPITO (CD analysis and plotting tool) server. ${ }^{31}$

\section{Comparative Analyses of the Drug-Like Properties of LFA-9 and Licofelone}

The Lipinski rule of five helps in distinguishing between drug-like and non-drug-like molecules. Lipinski's rule of five states that most drug-like molecules have:- $\log \mathrm{P} \leq 5$; molecular weight $\leq 500$; number of hydrogen bond acceptors $\leq 10$; and number of hydrogen bond donors $\leq 5 .{ }^{32}$ In addition, the number of rotatable bonds are also calculated to determine their bioavailability. ${ }^{33,34}$ Molecules violating more than one of these rules may have problems with bioavailability. The drug-like properties of LFA-9 and licofelone were calculated using a molinspiration tool. ${ }^{32}$

\section{In vitro Metabolic Stability of LFA-9 Was} Assessed on S9 Fraction of Mouse/Rat

\section{Liver}

In vitro metabolic stability of LFA-9 was assessed on S9 fraction of mouse/rat liver activated with glutathione, UDPglucuronic acid, NADPH, PAPS (sulfonation). ${ }^{35} \mathrm{~S} 9$ fraction of mouse/rat liver was isolated and further activated with NADPH (40 mM), UDPGA $(20 \mathrm{mM})$, and GSH $(2 \mathrm{mM})$, respectively while that of PAPS $(2 \mathrm{mg} / \mathrm{mL})$ before treatment with LFA-9 and licofelone $(500 \mu \mathrm{M})$ at different time intervals $(0,15,30,45$, and 60 minutes). Further, the compound was extracted using acetonitrile and quantified using HPLC. Percent of remaining compound was calculated by using the following formula: (Peak area at $0 \mathrm{~min}$ - Peak area at 60 $\mathrm{min} /$ Peak area at $0 \mathrm{~min}) \mathrm{x} 100$.

\section{Colon Tumor Spheroid Culture Using $\mathrm{Apc}^{\text {Pirc/+ }}$ (Pirc) Rats}

Isolated crypts or single cells were isolated from colorectum of $A p c^{\text {Pirc/+ }}$ rats and cultured as previously described. ${ }^{36}$ Briefly, crypts or single cells were plated in ultra-low attachment wells of 24-well plates. After cell seeding, $500 \mu \mathrm{L}$ of culture medium containing $1 \%$ B27/ N2, 1\% FBS, EGF (50 ng/mL), Noggin (100 ng/mL), Rspondin $1(500 \mathrm{ng} / \mathrm{mL})$, and $\mathrm{Y}-27632(10 \mu \mathrm{M})$ was added. Cell culture medium was changed every other day. Spheroids were counted after 14 days. The results were expressed as average number of spheroids. The $\mathrm{PGE}_{2}$ levels in culture supernatant in treated and untreated wells were estimated using $\mathrm{PGE}_{2}$ ELISA kits (Cayman Chemical). The results were presented in $\mathrm{pg} / \mathrm{mL}$.

\section{MTT Assay}

MTT assay was performed to assess the cytotoxic effect of compounds (LFA-9 and licofelone) on normal colonocytes of rats. ${ }^{37}$ Cells were treated with compounds at various concentrations $(0.1-100 \mu \mathrm{M})$ for 24 hours. Then, $20 \mu \mathrm{L}$ of freshly prepared MTT reagent was added to cells and incubated at $37^{\circ} \mathrm{C}$ for 2 hours. The supernatant growth medium was removed and replaced with DMSO (100 $\mu \mathrm{L})$ to dissolve the formazan crystals. The optical density (absorbance) was read using a microplate reader at $570 \mathrm{~nm}$ with a reference wavelength of $620 \mathrm{~nm}$. Three individual experiments were performed and results were expressed as percent viability at various doses of each compound.

\section{In vivo Anti-Inflammatory Activity by Carrageenan-Induced Rat Paw Edema Model (Animal Studies)}

The carrageenan-induced paw edema model is the most widely used for the evaluation of anti-inflammatory activity. ${ }^{38}$ Male Fischer (F344) rats weighing 350-400 g were housed under standard conditions (temperature of $21^{\circ} \mathrm{C}$ with an alternating 12 -h light-dark cycle and humidity of $50 \%$ and 20 air changes per hour, before and also during the experiment. The animals were fed with American Institute of Nutrition (AIN) modified-76A standard laboratory diet. During the experiment, the rats were allowed water and food ad libitum. Animal experimental protocol was approved by Institutional Animal Care and 
Use Committee at the University of Oklahoma Health Sciences Centre (Protocol \# 18-104-FH). Animal experiments were conducted according to the Guide for the Care and Use of Laboratory Animals (National Research Council of the National Academies) and as per the Animal Welfare $\mathrm{Act}^{39}$ (https:/www.nal.usda.gov/awic/ani mal-welfare-act).

The animals were divided into three groups: Group I served as control (carrageenan), group II (saline), group III (LFA-9400 ppm) and group IV (licofelone 400 ppm). The animals were fed with AIN-76A diet containing control or LFA-9/licofelone (400 ppm) and injected (subcutaneous) $0.1 \mathrm{~mL}$ of $1 \% \lambda$-carrageenan solution with saline into subplantar region of left hind paw of each rat. The right hind paw of the same rat was treated with $0.1 \mathrm{~mL}$ of saline alone in the same manner as the control. Before induction of edema, the dorsiventral thickness (millimeters (mm)) of both the paws of each was measured using digital vernier caliper and also the measurements were taken at 1-, 2-, 3-, 4-, 5- and 6-hours after carrageenan injection. The results are expressed in percent edema inhibition. The stomach and other major organs were examined for ulcers and any other toxicity. ${ }^{40}$ The paw exudates were collected and used for $\mathrm{PGE}_{2}, \mathrm{LTB}_{4}, \mathrm{TXB}_{2}$ and $\mathrm{PGI}_{2}$ quantifications using ELISA kits (Cayman Chemical, MI, USA).

\section{Immunofluorescence Technique}

CD68-positive inflammatory cell infiltration and TNF- $\alpha$ expression were evaluated using immunofluorescence technique on the paw tissue sections. ${ }^{38}$ Heat-induced epitope retrieval was performed on $4-\mu \mathrm{m}$ formalin-fixed, paraffin-embedded sections in citrate buffer $(\mathrm{pH} 6.0)$ at $99^{\circ} \mathrm{C}$ for 30 minutes. Slides were incubated in a normal serum and BSA blocking step at room temperature for 40 minutes. After incubation with primary antibody [Alexa Fluor 700-conjugated CD68 (1:50 dilution) (Novus Biologicals (NB600985)) and TNF- $\alpha$ (1:100 dilution) (Santa Cruz)] overnight at $4{ }^{\circ} \mathrm{C}$, slides were labeled with Alexa Fluor 488 dye-conjugated secondary antibody (for TNF- $\alpha$ ) and mounted with ProLong Gold (Thermo Fisher Scientific). Further, the slides were examined utilizing SP8 confocal microscopy (Leica). MetaMorph Microscopy Automation and Image Analysis Software was used to quantify the cell number with fluorescent staining.

\section{Immunoblot Analysis}

Twenty-micrograms of the total protein was size-separated in a $12 \%$ SDS-polyacrylamide gel and transferred electrophoretically onto a PVDF membrane using a semidry blot transfer apparatus (Bio-Rad, Hercules, CA, USA). Further, the membrane was blocked and incubated overnight with a primary antibody (CD68 (1: 100 dilution) (Santa Cruz, Dallas, TX, USA) and TNF- $\alpha$ (1:100 dilution)) and was subsequently incubated with horseradish peroxidase-conjugated secondary antibody. The proteins were detected using ECL reagents (Biorad). Beta-actin was used as the loading control. ${ }^{38}$

\section{Statistical Analysis}

Each experiment was conducted in triplicate and expressed as mean \pm standard deviation. Inhibitory Concentration (IC) 50 values were calculated based on the dose-response curve vs log inhibitor concentration through variable slope (four parameters) of non-linear regression analysis using GraphPad prism software. Statistical differences between control and treated groups were evaluated using unpaired $t$ test with Welch's correction. $P$-values $\leq 0.05$ for data sets were considered significant. Significance of the results are depicted in all graphs and tables as not significant (NS), $(P>0.05) ; *(P \leq 0.05) ; * *(P \leq 0.01) ; * * *(P \leq 0.001) ; * * * *$ $(P \leq 0.0001)$.

\section{Results}

\section{In silico Screening for Identification of mPGES-I and 5-LOX Inhibitor LFA-9}

A hit library of mPGES-1 inhibitors and its active fragments $(\sim 1000)$ was prepared from BRENDA and NCI databases based on Lipinski's rule of 3. To remove possible false hits, an online program PAINS Remover (panassay interfering compound substructures http://cbligand. org/PAINS) was used to screen all the designed licofelone analogues and found that all these analogues passed this test (Supplemental Information Table 1 and 2). ${ }^{41,42}$ The licofelone pharmacophore (the lead molecule) was optimized at its carboxylic group using different hit fragments through rational drug design strategy to target mPGES-1/ 5-LOX while sparing COX-1/2 activities of human, mouse, and rat (Supplemental Information Table 1 and 2). The licofelone derivatives were designed to target mPGES-1 and 5-LOX of human, mouse, and rat for their pre-clinical development and clinical translation. Among all designed licofelone derivatives (Supplemental Information Table 2), the addition of 2-amino acetic acid (glycine) at the carboxyl moiety of licofelone (a lead molecule LFA-9) led to strong binding interactions with 
active site of human, mouse, and rat mPGES-1 and 5LOX, but exhibited poor interactions with COX-1/2 (Figure 2). Moreover, LFA-9 obeys Lipinski`s rule of 5 for drug-likeness, but licofelone does not (Supplemental Information Table 3 ). The calculated $\log P$ value of licofelone is 6.15 (more than 5).

LFA-9 strongly bound with human, mouse, and ratspecific mPGES-1 with binding energies ranging between -238.27 and $-257.76 \mathrm{Kcal} / \mathrm{mol}$. LFA-9 showed strong interactions with the key amino acid residues of the mPGES-1 active site (Figure 2A; Table 1). mPGES1 (trimer) forms active site pockets with polar residues like Arg126 for conversion of $\mathrm{PGH}_{2}$ to $\mathrm{PGE}_{2}{ }^{43}$ LFA-9 formed hydrogen bond interactions with Asn74 and Arg126 of the mPGES-1. The extended -COOH group lies within proximity of Asn74 which acts as an h-bond donor and forms conventional hydrogen bond interactions with one of the oxygens $(=\mathrm{O})$ of the carboxylic acid moiety. Arg126 forms the hydrogen bond interactions with oxygen atom of the glycine residue. This interaction is very important as Arg126 is reported to be crucial for the catalysis of mPGES-1, and its mutation caused loss in mPGES-1 enzymatic activity. ${ }^{43-45}$ Apart from this a pi-donor hydrogen bond was also observed with Thr131 (similar to licofelone). Another crucial observation in the case of LFA-9 was the hydrophobic interactions formed with Tyr130. The Tyr130 formed strong pi-pi T-shaped interactions with the central 5-membered ring along with pi-alkyl interactions. Other hydrophobic interactions observed included alkyl with Pro124 and Val128. These amino acids are key for mPGES-1 catalytic activity in a previous study by Gupta et al. ${ }^{46}$ An atomic level interaction study demonstrated that LFA-9 formed hydrogen bonds and hydrophobic interactions with active site of amino acids mPGES-1 after inclusion of 2-aminoacetic acid at carboxyl end of the licofelone. The addition of 2-aminoacetic acid (glycine) before the terminal $-\mathrm{COOH}$ group showed an augmentation in the nature and strength of the interactions observed at the mPGES-1 active site. LFA-9 showed similar interactions with human, mouse and rat mPGES-1. These interaction analysis results clearly show the reason for improved mPGES-1 inhibitory efficacy of LFA-9 as compared to licofelone (Figure 2A; Table 1).

LFA-9 bound with 5-LOX (human/mouse/rat) with binding energies ranging between -199.85 and $-215.3 \mathrm{Kcal} / \mathrm{mol}$, which is comparable to that of licofelone. LFA-9 interacted with key active site amino acid Tyr181 of the 5-LOX and interferes with the iron that is coordinated by three conserved histidine residues (His 367, 372, and 550), as well as the main-chain carboxylate of the $\mathrm{C}$ terminus (Ile673) as part of the active site. ${ }^{47}$ LFA-9 and licofelone are interacting in the similar vicinity of 5-LOX. Addition of 2-aminoacetic acid before the terminal -COOH group of licofelone (LFA9) retains its 5-LOX inhibitory potential (Figure 2B). LFA-9 also interacts with His432 that helps to define the active site of 5-LOX ${ }^{47}$ (Figure 2B). However, LFA-9 shows poor binding interactions with COX-1 and COX-2 (Figure 2C and D). Licofelone interacts with key active site amino acids of COX-1 (Arg119) and COX-2 (Ser530) ${ }^{48,49}$ (Figure 2C and $\mathrm{D})$. The addition of 2-aminoacetic acid before the terminal -COOH group of licofelone (LFA-9) led to loss of these interactions with $\mathrm{COX}-1 / 2$ (sparing effect).

\section{Chemical Synthesis and Characterization of MPGES-I and 5-LOX Inhibitor LFA-9}

LFA-9 (2-[[2-[2-(4-chlorophenyl)-6,6-dimethyl-1-phenyl5,7-dihydropyrrolizin-3-yl]acetyl] amino]acetic acid) was synthesized in a two-step organic synthesis process and purity was found to be $>95 \%$ (Supplemental Figure 5A and $\underline{B}$ ). The retention time of LFA-9 is 6.7 minutes in HPLC analysis. Chemical structure of LFA-9 was assigned using advanced spectroscopic techniques, such as ${ }^{1} \mathrm{H},{ }^{13} \mathrm{C}$, and HRMS (Supplemental Figure 5C and $\underline{\mathrm{D}}$ ).

Yield: $80.5 \%$; Melting Point: $106-108^{\circ} \mathrm{C} .{ }^{1} \mathrm{H}$ NMR (300 $\left.\mathrm{MHz}, \mathrm{CDCl}_{3}\right) \delta$ (ppm): $1.29\left(\mathrm{~s}, 6 \mathrm{H}, 2 \times \mathrm{CH}_{3}\right), 2.85(\mathrm{~s}, 2 \mathrm{H}$, $\left.\mathrm{CH}_{2}\right), 3.55\left(\mathrm{~s}, 2 \mathrm{H}, \mathrm{CH}_{2}\right), 3.72\left(\mathrm{~s}, 2 \mathrm{H}, \mathrm{CH}_{2}\right), 4.04$ (d, Jgem=5.3 Hz, 2H, NH-CH$), 7.03-7.29(\mathrm{~m}, 9 \mathrm{H}, \mathrm{Ar}-\mathrm{H}) .{ }^{13} \mathrm{C}$ NMR (75 MHz, $\mathrm{CDCl}_{3}$ ) $\delta$ (ppm): 28.0, 33.0, 40.6, 41.2, 43.3, 58.0, 115.0, 117.2, 124.3, 124.8, 2×128.0, 128.5, 131.2, 131.9, 134.1, 134.9, 135.5, 171.4, 172.9; HRMS: m/z 437.1680 [M $+\mathrm{H}]^{+} ; 459.1508[\mathrm{M}+\mathrm{Na}]^{+}$, calcd. for 436.1680, found 437.1680 .

\section{5-LOX and mPGES-I Dual Inhibitory and COX-I/2 Sparing Effects of LFA-9 in Cell- Free Systems}

In a cell-free system, LFA-9 (Figure 3) substantially inhibited human, mouse, and rat mPGES-1 activities ( $\mathrm{IC}_{50}$ values: $0.87 \mu \mathrm{M}$ [human]; $0.52 \mu \mathrm{M}$ [mouse]; $1.40 \mu \mathrm{M}$ [rat]), as compared with its parental compound licofelone ( $\mathrm{IC}_{50}$ values: $14.04 \mu \mathrm{M}$ [human]; $>15 \mu \mathrm{M}$ [mouse]; $12.3 \mu \mathrm{M}$ [rat]). LFA-9 showed comparable 5LOX inhibitory activity ( $\mathrm{IC}_{50}$ values: $2.75 \mu \mathrm{M}$ [human]; 


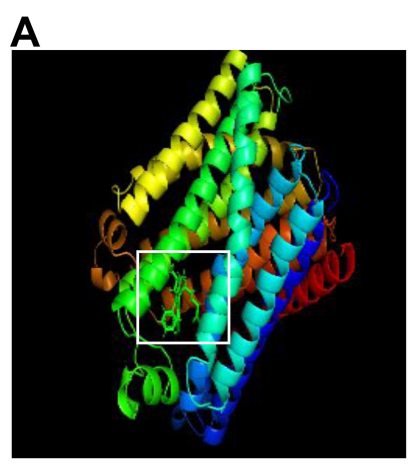

(i)

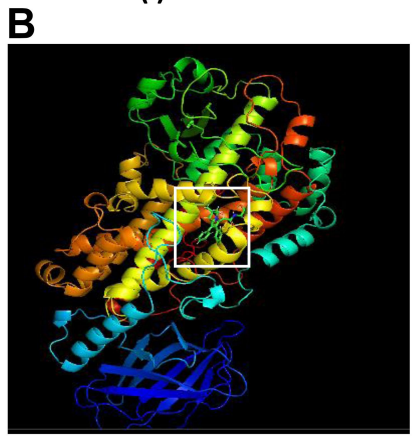

(i)

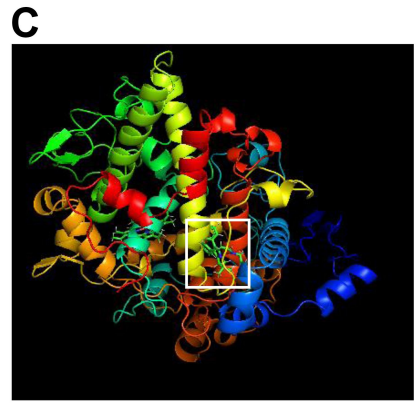

(i)

D

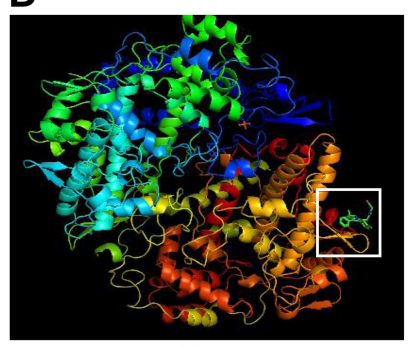

(i)
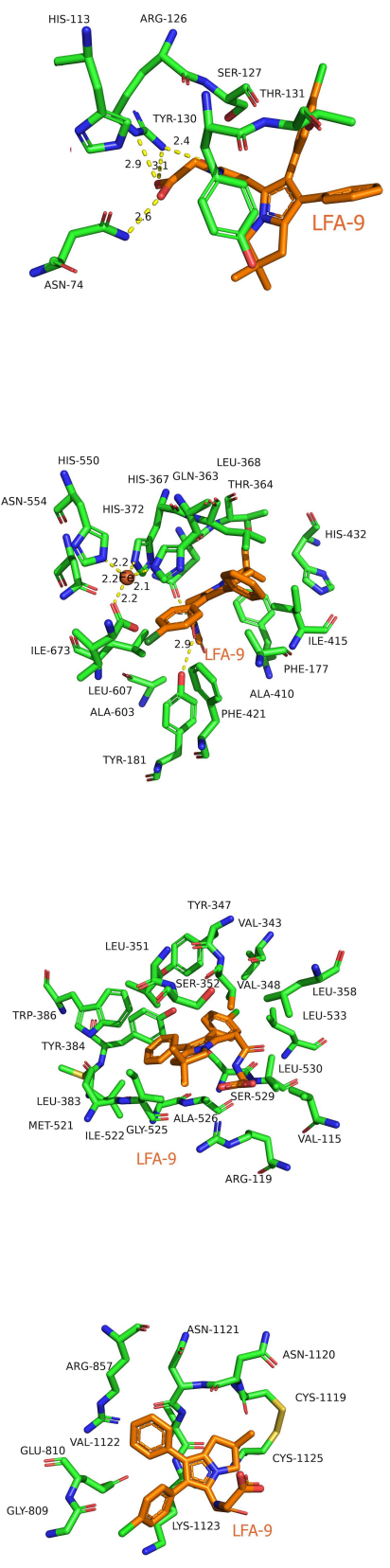
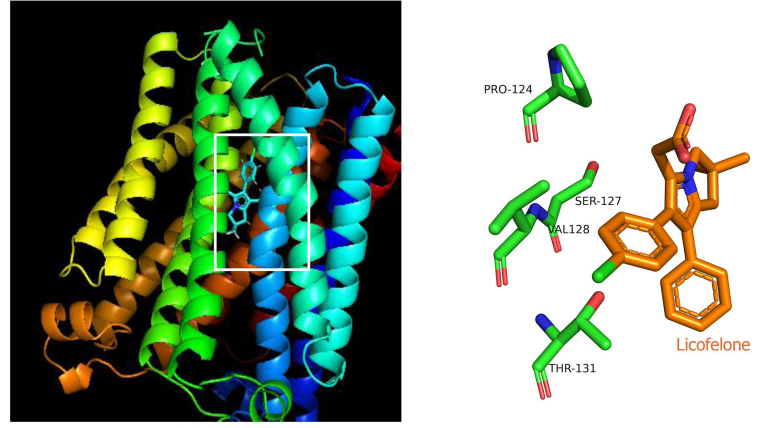

(ii)
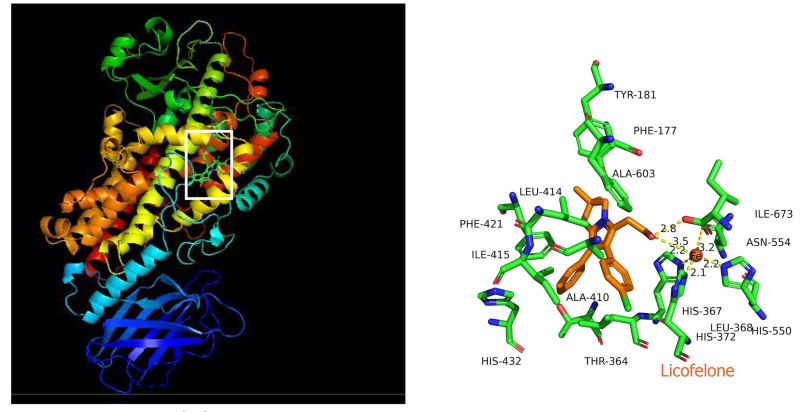

(ii)
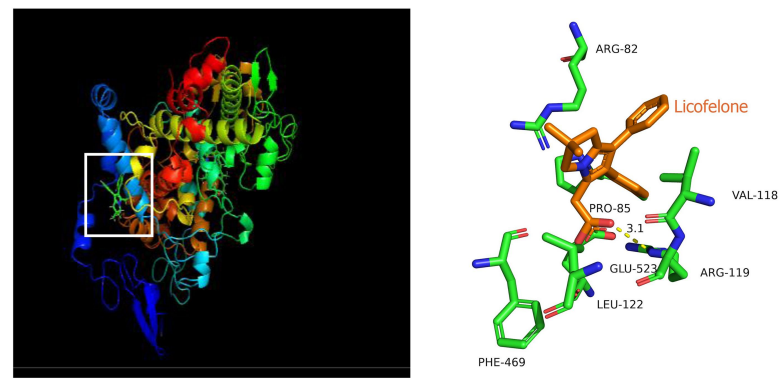

(ii)

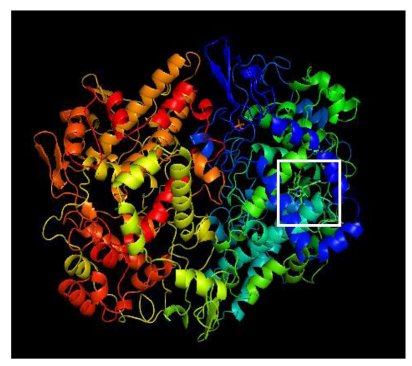

(ii)

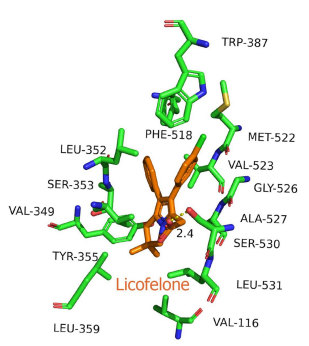

Figure 2 Molecular docking studies represent binding interaction of LFA-9 (i) with three dimensional structures of mPGES-I (A) (PDB ID: 4YL3), 5-LOX (PDB ID: 3O8Y) (B), COX-I (modelled) (C), and COX-2 (PDB ID: 5KIR) (D) in comparison with licofelone (ii). Highlighted square area in white color (ribbon model) represented the interacting amino acids in three-dimensional structures.

$2.64 \mu \mathrm{M}$ [mouse]; $0.89 \mu \mathrm{M}$ [rat]) to licofelone $\left(\mathrm{IC}_{50}\right.$ values: $2.13 \mu \mathrm{M}$ [human]; $2.34 \mu \mathrm{M}$ [mouse]; $0.64 \mu \mathrm{M}$ [rat]). Licofelone inhibited COX-1 ( $\mathrm{IC}_{50}$ values: 1.65 $\mu \mathrm{M}$ [human]; $1.74 \mu \mathrm{M}$ [mouse]; $1.81 \mu \mathrm{M}$ [rat]) and
COX-2 ( IC $_{50}$ values: $1.36 \mu \mathrm{M}$ [human]; $2.01 \mu \mathrm{M}$ [mouse; $3.26 \mu \mathrm{M}$ [rat]), but LFA-9 did not show inhibition of human, mouse, or rat COX-(1and2) activity up to the tested range of $1 \mathrm{mM}$ (Figure 3). 
Table I Binding Energies of LFA-9 on mPGES-I, COX-I/2, and 5-LOX

\begin{tabular}{|l|l|l|l|}
\hline \multirow{2}{*}{ Enzyme } & \multirow{2}{*}{ Species } & \multicolumn{2}{|l|}{ Binding Energy (Kcal/mol) } \\
\cline { 3 - 4 } & & Licofelone & LFA-9 \\
\hline 5-LOX & Human & -208.58 & -199.85 \\
& Rat & -212.36 & -209.8 \\
& Mouse & -216.74 & -215.3 \\
\hline \multirow{2}{*}{ COX-I } & Human & -198.37 & -56.91 \\
& Rat & -192.52 & -66.28 \\
& Mouse & -188.56 & -63.33 \\
\hline \multirow{2}{*}{ COX-2 } & Human & -213.2 & -85.31 \\
& Rat & -205.37 & -72.42 \\
& Mouse & -211.53 & -66.54 \\
\hline \multirow{2}{*}{ mPGES-I } & Human & -104.35 & -245.34 \\
& Rat & -135.25 & -257.76 \\
& Mouse & -142.63 & -238.27 \\
\hline
\end{tabular}

\section{LFA-9 Shows Strong Binding Affinity} Causes Unfolding of the Secondary Structures of Human mPGES-1/5-LOX

To validate results from molecular docking studies, binding studies of LFA-9 and mPGES-1 and 5-LOX were performed using ITC, and found strong binding affinity based on accurate fitting of the isotherm. The KD (dissociation constant) values of LFA-9 for human and rat mPGES-1 were 2.1 and $3.3 \mu \mathrm{M}$, respectively (Table 2, Supplemental Figure 6). Human 5-LOX had two KDs, $18 \mu \mathrm{M}$ and $210 \mu \mathrm{M}$, indicating two binding sites (Table 2, Supplemental Figure 6). This finding provides evidence that LFA-9 interacts with mPGES-1 at one binding site, while LFA-9 interacts with the 5-LOX enzyme at two binding sites. Further, the effect of LFA-9 on the secondary structure of mPGES-1, COX-1 and 2, and 5-LOX was evaluated using the circular dichroism (CD) technique. The interaction of LFA-9 (selected dose at $0.12 \mu \mathrm{M})$ with the secondary structure of mPGES-1 was determined based on the shift in bands at $208 \mathrm{~nm}$ and $220 \mathrm{~nm}$ of the CD spectrum, which is indicative of a $19 \%$ loss of $\alpha$-helix content (Table 3, Supplemental Figure 7). This result suggested that the decrease in $\alpha$-helix content of mPGES-1 led to its partial unfolding, which resulted in inhibition of its enzyme activity. LFA-9 binds strongly and unfolds the $\alpha$-helix $(8.7 \%)$ and $\beta$-sheets (1\%) of 5-LOX at $0.1 \mu \mathrm{M}$, thereby inhibiting 5-LOX activity (Table 3, Supplemental Figure 7).

\section{$\mathrm{PGE}_{2}$ and $\mathrm{LTB}_{4}$ Inhibitory, and $\mathrm{PGI}_{2}$ Sparing Effects of LFA-9 in a Human/ Mouse/Rat Cell-Based System}

In cell-based assays (Figure 4), the effect of LFA-9 on $\mathrm{PGE}_{2}, \mathrm{LTB}_{4}$, and $\mathrm{PGI}_{2}$ production was evaluated in LPS-stimulated macrophages of humans, mice, and rats, and compared with that of licofelone. LFA-9 inhibited $\mathrm{PGE}_{2}\left(\mathrm{IC}_{50}\right.$ range $\left.0.47-0.78 \mu \mathrm{M}\right)$ and $\mathrm{LTB}_{4}\left(\mathrm{IC}_{50}\right.$ range $0.63-1.24 \mu \mathrm{M})$ production and spared $\mathrm{PGI}_{2}\left(\mathrm{IC}_{50}\right.$ $>100$ ) in LPS-stimulated macrophages of humans, mice, and rats, whereas licofelone inhibited production of $\mathrm{PGE}_{2} \quad\left(\mathrm{IC}_{50}\right.$ range $\left.0.56-1.31 \mu \mathrm{M}\right), \mathrm{LTB}_{4}\left(\mathrm{IC}_{50}\right.$ range $0.53-1.6 \mu \mathrm{M})$, and $\mathrm{PGI}_{2}\left(\mathrm{IC}_{50}\right.$ range $\left.0.85-0.91 \mu \mathrm{M}\right)$. Unlike licofelone, LFA-9 effectively inhibited $\mathrm{PGE}_{2}$ and $\mathrm{LTB}_{4}$ production in LPS-stimulated macrophages of humans, mice, and rats, with no effect on $\mathrm{PGI}_{2}$ production (Figure 4).

\section{LFA-9 Inhibits Inflammogen (Carrageenan)-Induced Hind Paw Edema of Rats}

In vivo oral anti-inflammatory efficacy of LFA-9 (400 ppm) was tested on a group of rats $(n=3)$ using carrageenan model and found $70.4 \%$ edema inhibition (maximum) at 3 hours after carrageenan injection, whereas licofelone (400 ppm) showed $61.7 \%$ edema inhibition (Figure 5A and B). LFA-9 (400 ppm) did not show any gastrointestinal toxicity (Supplemental Figure 8; Supplemental Information Table 4). LFA-9 reduced $\mathrm{PGE}_{2}(P \leq 0.0001)$, and $\operatorname{LTB}_{4}(P \leq$ 0.01) levels but spared $\mathrm{PGI}_{2}$ in paw exudates compared to control (vehicle), while, licofelone reduced $\mathrm{PGE}_{2}, \mathrm{TXB}_{2}$, $\mathrm{LTB}_{4}$, and $\mathrm{PGI}_{2}$ levels. LFA-9 maintained homeostasis of $\mathrm{TXB}_{2}$ and $\mathrm{PGI}_{2}$ production levels as that of control (saline). This study demonstrates the anti-inflammatory potential of LFA-9 by inhibiting production of $\mathrm{PGE}_{2}$ and $\mathrm{LTB}_{4}$, while sparing $\mathrm{PGI}_{2}$ levels (Figure $5 \mathrm{C}$ ). Further, immunofluorescence and immunoblot analysis of paw tissue sections demonstrated that CD68-positive inflammatory cell infiltration and expression of TNF- $\alpha$ are significantly decreased in the LFA-9-treated group compared to the untreated control (Figure 5D-F). LFA-9 showed better efficacy in the reduction of CD68-positive inflammatory cell infiltration $(P \leq$ 0.0001 ) and suppression of TNF- $\alpha$ expression than licofelone (Figure 5D-F). 
A

(i)

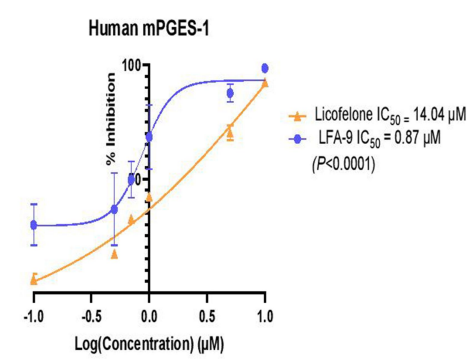

B

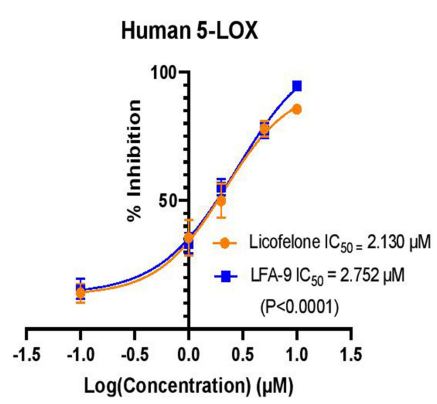

C

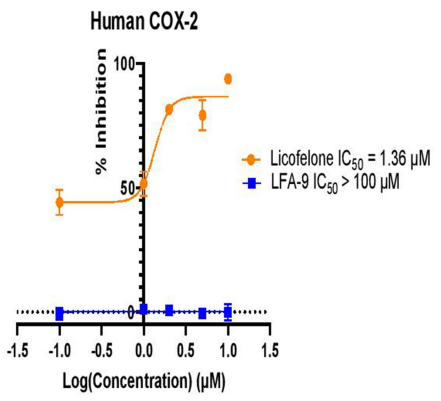

D

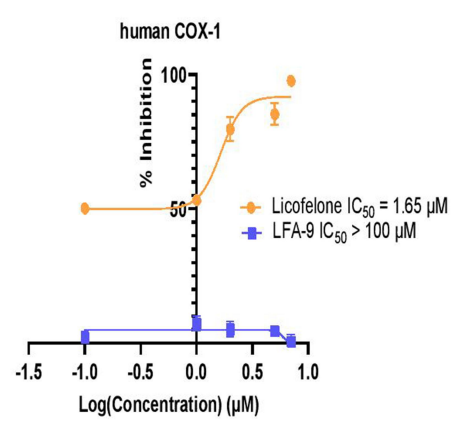

(ii)

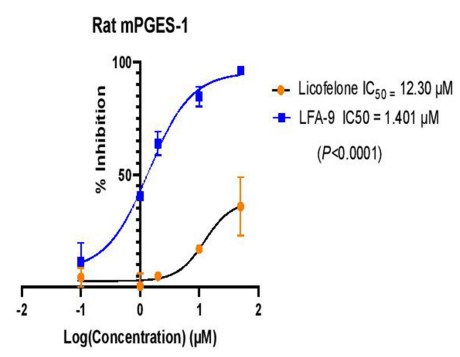

(ii)

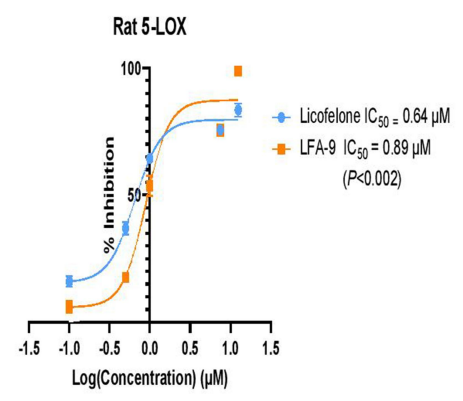

(ii)

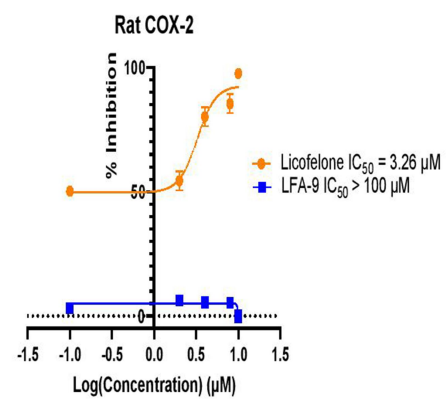

(ii)

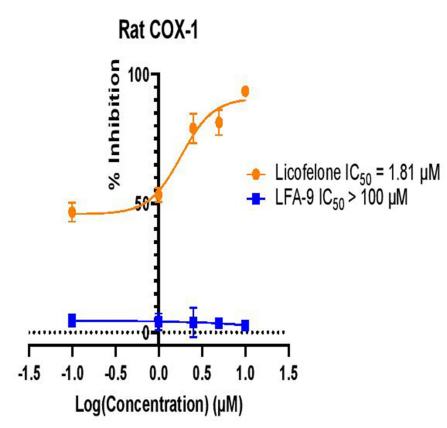

(iii)

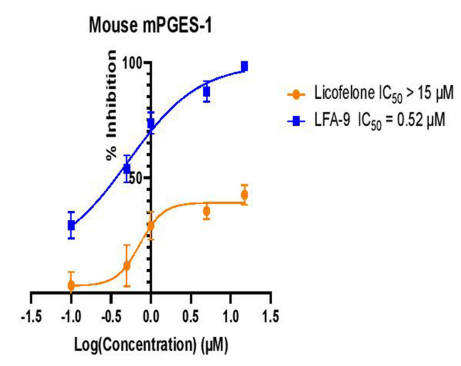

(iii)

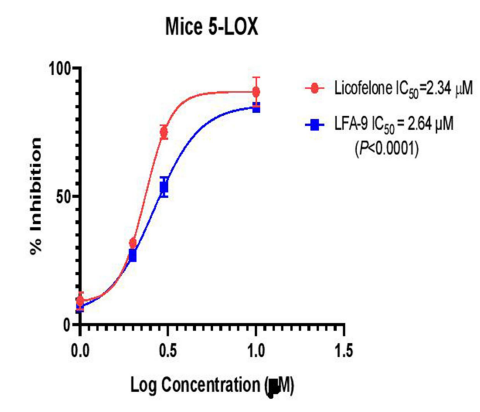

(iii)

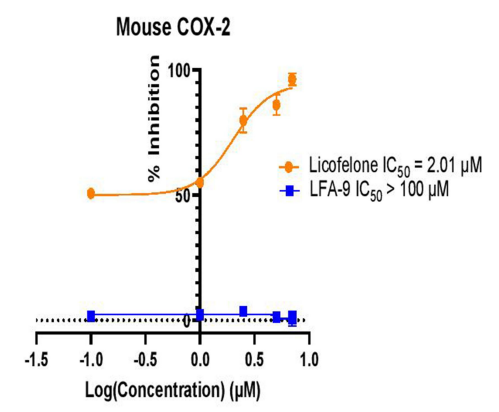

(iii)

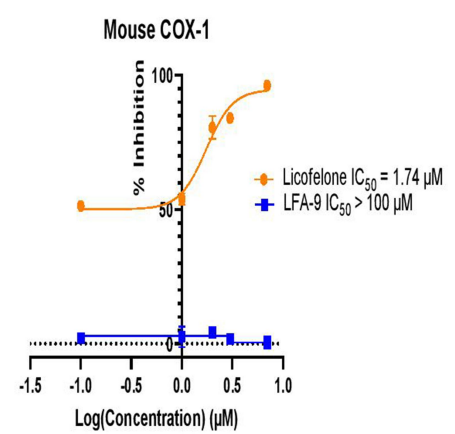

Figure 3 mPGES-I and 5-LOX dual inhibitory activity of LFA-9 in a cell-free system. (A). Effect of LFA-9 and licofelone on human (i), rat (ii), mouse (iii) specific mPGES-I (A), 5-LOX (B), COX-I (C), COX-2 (D) and on an in vitro cell-free system. IC 50 values were calculated based on the dose-response curve vs log inhibitor concentration though variable slope (four parameters) of non-linear regression analysis. 
Table 2 Binding Efficacy Representing KD Values of LFA-9 on Human mPGES-I, COX-I/2, and 5-LOX

\begin{tabular}{|l|l|}
\hline Type of Enzyme & KD \\
\hline Human 5-LOX (sequential binding sites) & $18 \mu \mathrm{M}(\mathrm{KDI})$ \\
\cline { 2 - 2 } & $210 \mu \mathrm{M}(\mathrm{KD} 2)$ \\
\hline Human COX-2 & $>1 \mathrm{mM}$ \\
\hline Human COX-I & $>1 \mathrm{mM}$ \\
\hline Human mPGES-I & $2.1 \mu \mathrm{M}$ \\
\hline Rat mPGES-I & $3.3 \mu \mathrm{M}$ \\
\hline
\end{tabular}

\section{LFA-9 Prevents Colorectal Tumor}

\section{Stemness and Spheroid Formation in vitro} LFA-9 significantly inhibited $60.2 \%$ of colorectal tumor spheroids at $25 \mu \mathrm{M}$ as compared with the untreated control, whereas licofelone inhibited only $22.3 \%$ of colonic spheroids at $25 \mu \mathrm{M}$ (Figure 6A). LFA-9 inhibited $82 \%$ of $\mathrm{PGE}_{2}$ production as compared with the untreated control, whereas licofelone inhibited $63 \%$ of $\mathrm{PGE}_{2}$ production (Figure 6B). Moreover, LFA-9 showed no cytotoxicity to normal rat colonic epithelial cells (Supplemental Information Figure 9). LFA-9 exhibited better efficacy in prevention of colon tumor stemness than licofelone.

\section{LFA-9 Metabolically Stable in Activated- S9 Fraction of Liver}

LFA-9 is metabolically stable in activated-S9 fraction of mouse/rat liver for the tested time (1 hour). HPLC analysis demonstrated that $98.3 \%$ and $96.1 \%$ of LFA-9 remains after 60-minutes incubation in rat and mouse liver S9 fraction, respectively (Supplemental Information Figure 10).

\section{Discussion}

Pharmacological inhibitors of mPGES-1/5-LOX are proposed as alternative and safer anti-inflammatory agents to
NSAIDs and COXIbs. ${ }^{5-7}$ Licofelone is a potent inhibitor of COX-1/2, 5-LOX, and weak inhibitor of mPGES- 1 with anti-inflammatory efficacy, but did not enter into the market due to COX-associated toxicities. ${ }^{14,15}$ Licofelone is an excellent scaffold for mPGES-1/5-LOX dual inhibitors development with COX-1/2 sparing effects. ${ }^{8}$ In this study, pharmacophore of licofelone was modified at its carboxylic end by addition of various active fragments of mPGES-1 inhibitors to enhance mPGES-1 inhibitory potential and spares COX-1/2 activity. Interestingly, the addition of 2-amino acetic acid (glycine) at carboxyl end of licofelone led to enhanced interactions with active site of mPGES-1 and loss of interactions with active sites of COX-1/2, but no significant change in its interactions with active site of 5-LOX. In vitro and in silico studies demonstrated that licofelone analog LFA-9 showed substantial 5LOX and mPGES-1 dual inhibitory activity and spared COX-1/2 activities in human, mouse, and rat-based systems. LFA-9 showed several folds higher mPGES-1 inhibitory activity than did its parental molecule licofelone. In structure activity relationship studies demonstrated that addition of 2-amino acetic acid at the carboxyl end of licofelone improved inhibition of mPGES-1 and 5-LOX, and spared COX-1/2 activity. CD and ITC-based studies demonstrated that LFA-9 binds and induces changes in the secondary structure of human mPGES-1, thereby inhibiting their enzyme activities, based on in vitro studies. Using LPS-stimulated human/mouse/rat macrophages as the cell-based system, LFA-9 inhibited $\mathrm{PGE}_{2}$ and $\mathrm{LTB}_{4}$ due to mPGES-1/5-LOX dual inhibitory efficacy, and spared $\mathrm{PGI}_{2}$ production due to its $\mathrm{COX}-1 / 2$ sparing effect. Moreover, LFA-9 was more effective in a cell-based system than a cell-free system. Results from in silico, in vitro, and ex vivo studies of LFA-9 that were performed using human/mouse/rat mPGES-1 and 5-LOX are useful to facilitate its pre-clinical development and clinical translation.

Moreover, LFA-9 is metabolically stable in activated rat/ mice hepatic $\mathrm{S} 9$ fraction in vitro for 60 minutes. Lipinski's rule

Table 3 Effect of LFA-9 on Secondary Structure of Human mPGES-I and 5-LOX

\begin{tabular}{|l|l|l|}
\hline Enzyme & Concentration of LFA-9 $(\boldsymbol{\mu M})$ & Change in Secondary Structure \\
\hline Human mPGES-I & 0.12 & $19 \%$ unfolding in $\alpha$-helix content \\
\hline Human COX-I & 10 & No change in secondary structure \\
\hline Human COX-2 & 10 & No change in secondary structure \\
\hline Human 5-LOX & 0.12 & Unfolding in (8.7\%) $\alpha$-helix and (I\%) $\beta$-sheets \\
\hline
\end{tabular}


A

(i)

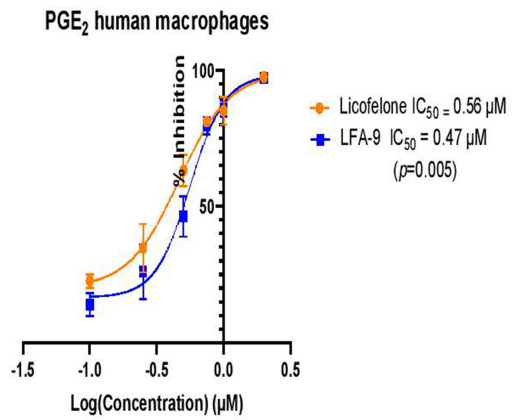

B

(i)

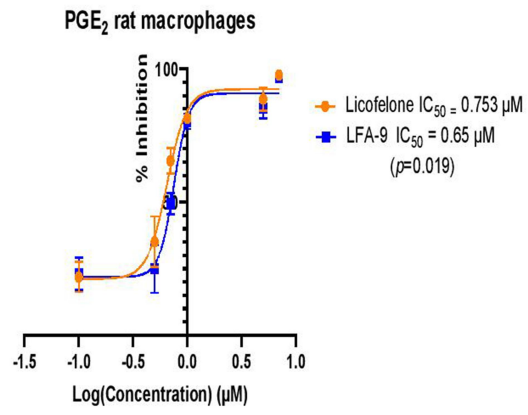

C

(i)

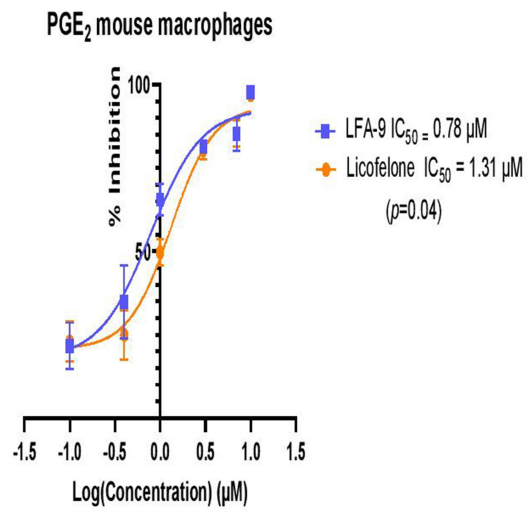

(ii)

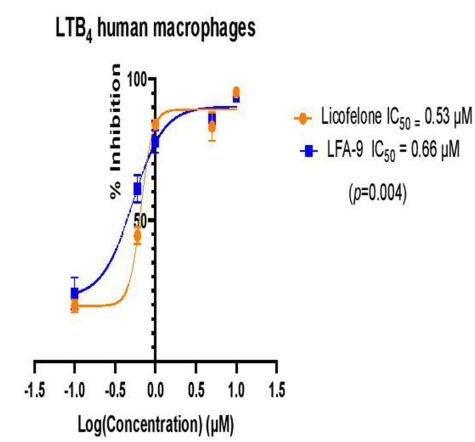

(ii)

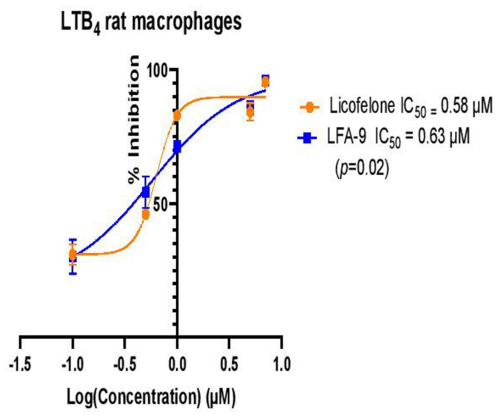

(ii)

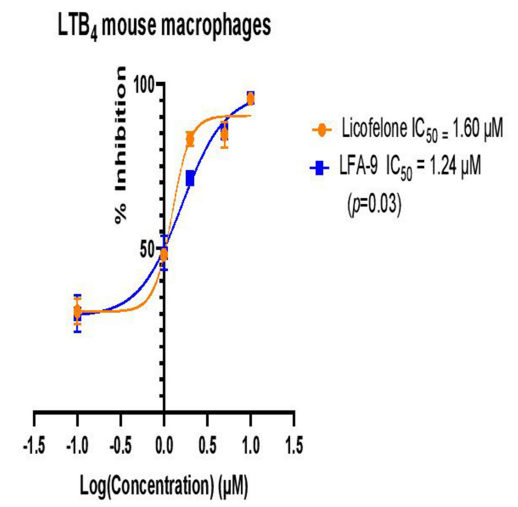

(iii)

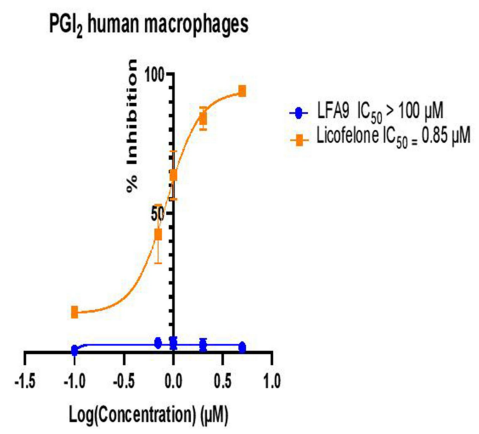

(iii)

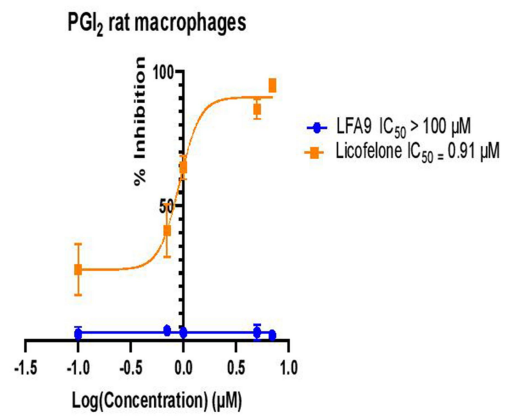

(iii)

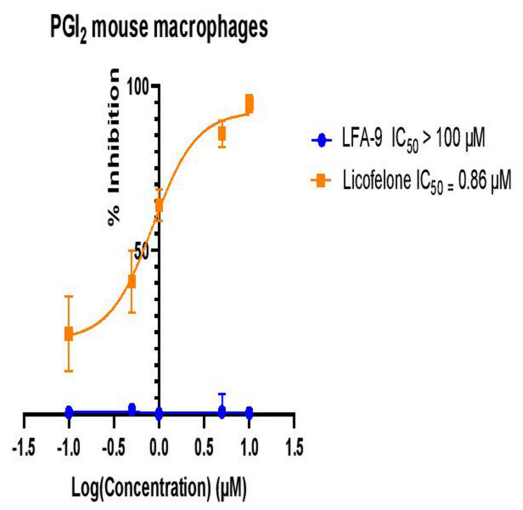

Figure $4 \mathrm{PGE}_{2}$ and $\mathrm{LTB}_{4}$ inhibitory, and PGI 2 sparing effects of LFA-9 in a cell-based system. Effect of LFA-9 on PGE 2 (i) and LTB 4 , (ii) and PGI 2 (iii) production in LPSstimulated human (A), rat (B), and mouse (C) macrophages (cell-based assays). IC $_{50}$ values were calculated based on the dose-response curve vs log inhibitor concentration though variable slope (four parameters) of non-linear regression analysis. $P$-values $\leq 0.05$ for data sets were considered significant.

of five and other properties like number of rotatable bonds (Nrotb) of any small molecules represent drug-likeness and demonstrate whether they are orally active molecules. ${ }^{33,34}$ Gupta et $\mathrm{al}^{34}$ described the importance of LogP and Nrotb along with dock scores in the discovery and development of
mPGES-1 inhibitors. Unlike licofelone, LFA-9 obeyed Lipinski's rule of 5 and number of rotatable bonds for druglikeness (Supplemental Information Table 3). Thus, LFA-9 may show better pharmacokinetic and dynamic properties than does licofelone. ${ }^{33,34}$ 


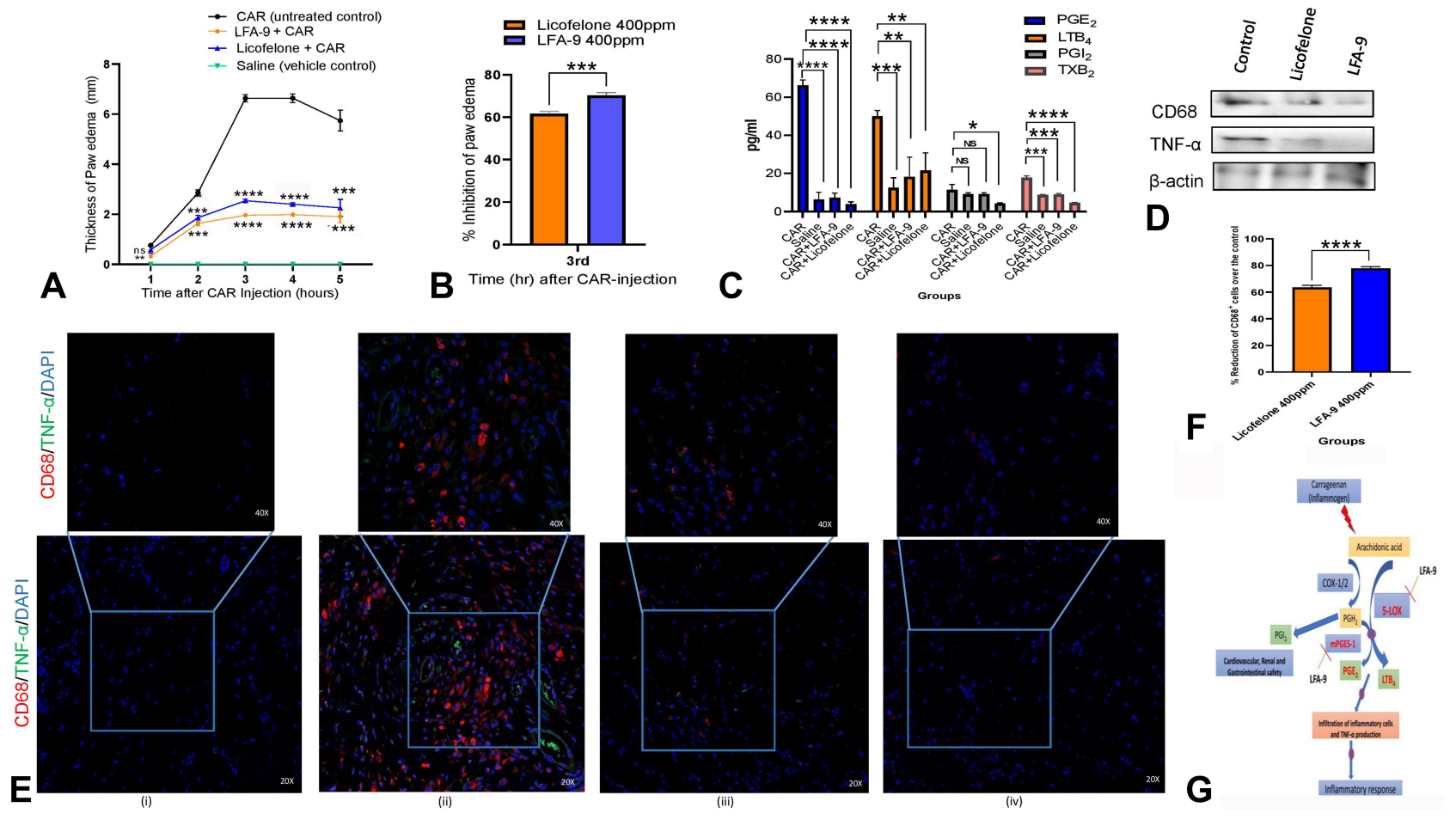

Figure 5 Anti-inflammatory activity of LFA-9. Paw thickness (A) and percent inhibition (B) using inflammogen (carrageenan (CAR))-induced rat paw edema. (C) Effect of LFA-9 on PGE 2 , LTB 4 , PGI 2 , and TXB 2 levels in exudates of carrageenan (CAR))-induced rat paw edema. (D) Effect of LFA-9 on expression of CD68 and TNF- $\alpha$ in CARinjected paw tissue using immunoblot analysis. (E and F) Effect of LFA-9 on CD68-positive inflammatory cell infiltration and TNF- $\alpha$ expression in CAR-injected paw tissue sections using immunofluorescence technique. $P$-values $\leq 0.05$ for data sets were considered significant. (NS) Not significant $(P>0.05)$; $*(P \leq 0.05)$; $* *(P \leq 0.0 \mathrm{I})$; $* * *(P \leq$ $0.001)$; $* * * *(P \leq 0.000 I)$. (G) Schematic representation of mechanism of action of LFA-9 for its anti-inflammatory activity with safety.

In the pre-clinical study using an inflammatory animal model, LFA-9 showed better anti-inflammatory activity than that of licofelone in carrageenan (inflammogen)induced rat paw edema model. LFA-9 showed maximum edema inhibition at 3 hours after carrageenan injection, which is referred to as the second phase of acute inflammation, in which CD68-positive inflammatory cells are accumulated, pro-inflammatory enzymes of arachidonic acid pathways and TNF- $\alpha$ are over-expressed. ${ }^{29,38,50}$ Results also demonstrate that LFA-9 inhibited inflammatory response by inhibiting pro-inflammatory $\mathrm{PGE}_{2}$ and chemotactic $\mathrm{LTB}_{4}$ levels and thereby, preventing the infiltration of CD68 positive macrophages and granular neutrophils along with TNF- $\alpha$ expression (Figure 5G).

NSAIDs induce gastric damage (ulcers and erosions) due to COX-1/2 inhibition, while mPGES-1 inhibitors show gastric mucosal safety due to COXs sparing effect. $^{40,51,52}$ Chattopadhyay et $\mathrm{al}^{52}$ found that aspirin $(180 \mathrm{mg} / \mathrm{kg})$ caused gastric toxicity with potential antiinflammatory efficacy. Ding et $\mathrm{al}^{18}$ found gastric toxicity of celecoxib and developed human/mouse specific mPGES-1 inhibitory benzylidenebarbituric acid derivatives as anti-inflammatory agents with GI safety. LFA-9 is a human/rat/mouse-specific mPGES-1/5-LOX inhibitor and maintains homeostatic levels of $\mathrm{TXB}_{2}$ and spared $\mathrm{PGI}_{2}$ levels as equal to that of the healthy control group (saline treated). But, licofelone reduced the levels of $\mathrm{TXB}_{2}$ and $\mathrm{PGI}_{2}$ compared to that of the healthy control group (saline treated) (Figure 5G). LFA-9 (400 ppm) did not show any gastrointestinal and any other toxicities in the rats (Supplemental Figure 8; Supplemental Information Table 4). Therefore, the results of the study demonstrated that LFA-9 can be a safer anti-inflammatory agent and warrants detailed studies for its clinical translation.

A pro-inflammation mediator, PGE2, promotes tumor stemness and spheroid formation towards colonic tumor initiation and progression. ${ }^{53}$ Threedimensional tumor spheroids in vitro mimic in vivo solid colonic tumors for anticancer drug screening. ${ }^{54}$ LFA-9 inhibited colonic tumor spheroid formation of $A P C^{\text {pirc }}$ rats (a model mimics colonic familial polyposis formation of humans). It had been well established that $\mathrm{PGE}_{2}$ production in the microenvironment regulates colon tumor stemness during tumorigenesis, progression, and invasion. Therefore, this study demonstrated the cancer-preventive 

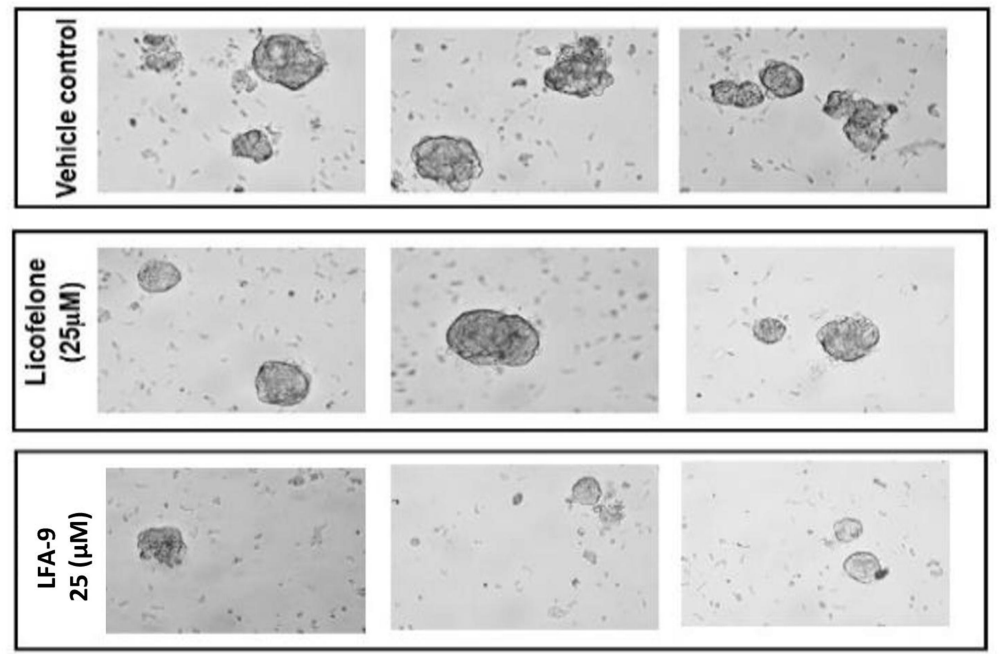

(i)

A

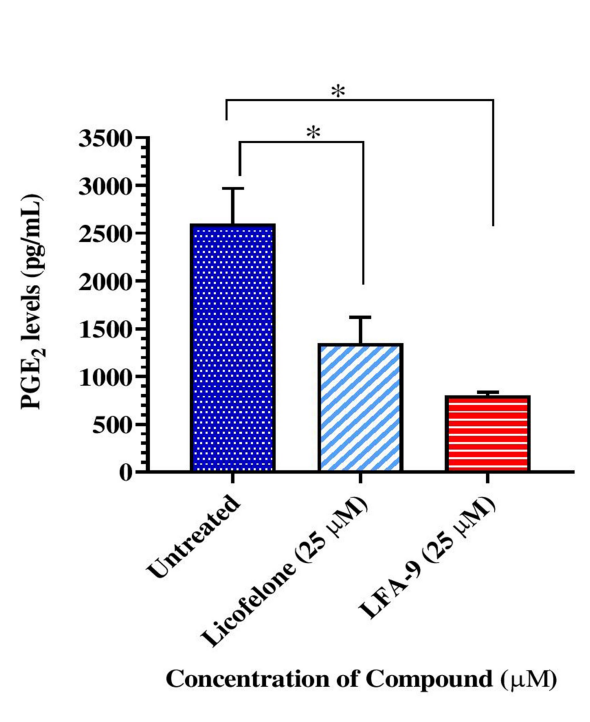

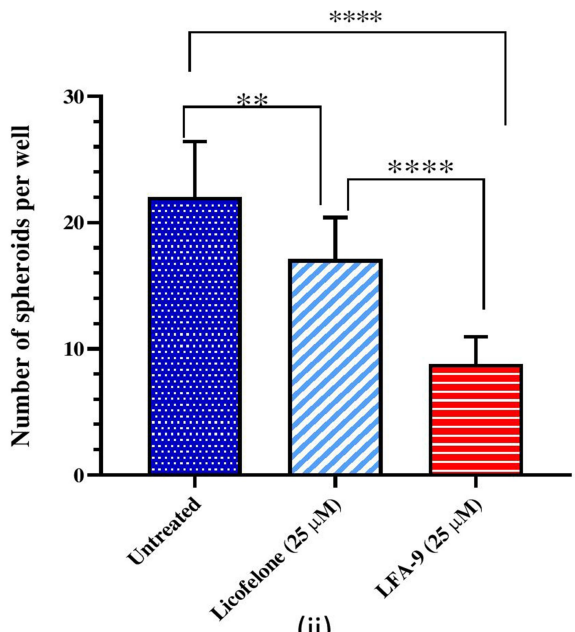

(ii)

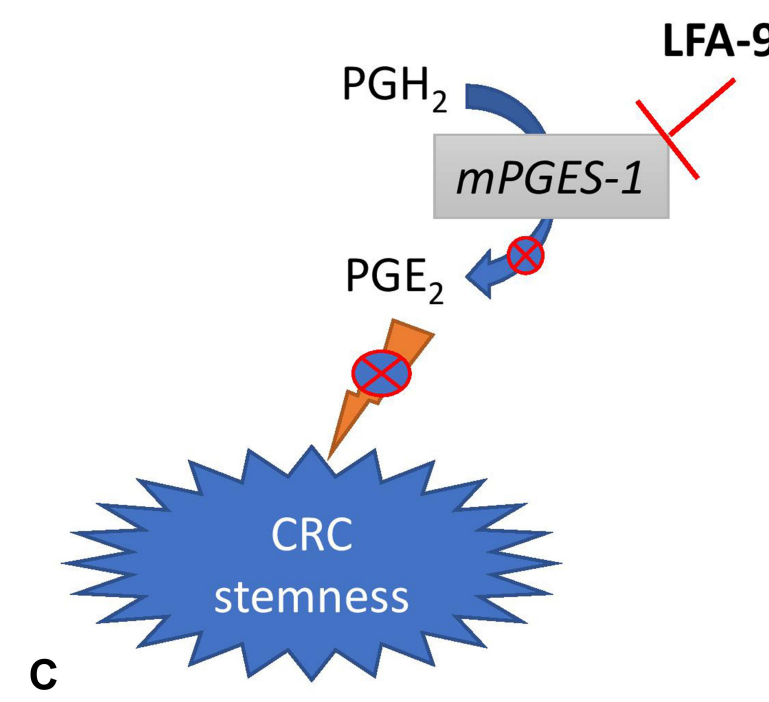

Figure 6 LFA-9 prevents colorectal cancer stemness. The effect of LFA-9 and licofelone on colon tumor stemness and colonic spheroids formation of APC ${ }^{\text {irc }}$ rats in vitro (A (i and ii)) and its inhibitory effect on PGE 2 production (B) in its culture supernatants. (C) Schematic representation of mechanism of action of LFA-9 for its preventive efficacy of colon tumor stemness. $P$-values $\leq 0.05$ for data sets were considered significant. Not significant $(P>0.05) ; *(P \leq 0.05)$; $* *(P \leq 0.0 \mathrm{I})$; $* * * *(P \leq 0.000 \mathrm{I})$.

effects of LFA-9 against tumorigenesis of CRC via its $\mathrm{PGE}_{2}$ inhibitory efficacy (Figure 6C). These results support our other study observations of LFA-9 inhibitory effects on $A P C^{\mathrm{min} /-}$ mice intestinal polyposis. ${ }^{25}$

\section{Conclusions}

In silico and in vitro (cell-free and cell-based) studies demonstrated that LFA-9 is a potent mPGES-1/5-LOX dual inhibitor by inhibiting products $\mathrm{PGE}_{2}$ and $\mathrm{LTB}_{4}$, respectively, and spares COXs and its downstream product $\mathrm{PGI}_{2}$. LFA-9 showed better druggable properties than did licofelone. In vivo studies demonstrated that LFA-9 showed substantial anti-inflammatory activity by inhibiting inflammogen (carrageenan)-induced paw edema in rats. LFA-9 prevented colon tumor stemness and spheroids formation in vitro, mediated by its $\mathrm{PGE}_{2}$ inhibitory effects. Therefore, mPGES-1/5-LOX inhibitor LFA-9 may be a new generation anti-inflammatory agent and the results warrant further pre-clinical studies on LFA-9 against several inflammatory diseases, including cancer.

\section{Abbreviations}

LFA-9, licofelone analogue-9; COX, cyclooxygenase; 5LOX, 5-lipoxygenase; mPGES-1, microsomal prostaglandin $\mathrm{E}$ synthase-1; CD, circular dichroism; $\mathrm{IC}_{50}$, inhibitory concentration 50; ITC, isothermal titration calorimetry; LTs, leukotrienes; $\mathrm{PGI}_{2}$, prostacyclin; $\mathrm{TXB}_{2}$, thromboxane $\mathrm{B}_{2} ; \mathrm{PGE}_{2}$, prostaglandin $\mathrm{E}_{2} ; \mathrm{CAR}$, carrageenan. 


\section{Data Sharing Statement}

The data sets and supporting data are available from the corresponding author upon reasonable request.

\section{Ethics Approval and Consent}

Animal experimental protocol (Protocol \# 18-104-FH) was approved by Institutional Animal Care and Use Committee at the University of Oklahoma Health Sciences Center.

\section{Acknowledgments}

The authors acknowledge NIH/NCI R01CA 213987, NIH/ NCI HHSN261201500024I and VA merit for funding support. The funders did not have any role in the design of the present study, data collection, data analysis, interpretation, or the writing of the manuscript. We thank the Laboratory for Molecular Biology and Cytometry Research and biochemistry core facility (isothermal titration calorimeter and circular dichroism spectropolarimeter services) at OUHSC for the use of the Core Facility, which provided HRMS service. N.S. Y. is thankful to Anil Singh, Yuting Zhang, Antony Bao and Guptha for their help during conduction of the experiments. The authors are grateful to the Laboratory of Biomolecular Structure and Function, OUHSC for providing the required facilities to perform protein purification and molecular modeling studies. We would also like to acknowledge the Laboratory for Molecular Biology and Cytometry Research for SP8 confocal microscopy services and data analysis.

\section{Disclosure}

Gopal Pathuri reports a patent US10206904B2 issued and a patent WO2017/015013 pending. Hariprasad Gali reports a patent US Patent 10206904 issued. Chinthalapally V. Rao reports a patent US10206904B2 issued and a patent WO2017/015013 A1 pending. The authors report no other potential conflicts of interest for this work.

\section{References}

1. White WB. Cardiovascular effects of the cyclooxygenase inhibitors. Hypertension. 2007;49(3):408-418. doi:10.1161/01. HYP.0000258106.74139.25

2. Brater DC, Harris C, Redfern JS, Gertz BJ. Renal effects of COX-2selective inhibitors. Am J Nephrology. 2001;21(1):1-15. doi:10.1159/ 000046212

3. Martel-Pelletier J. Therapeutic role of dual inhibitors of 5-LOX and COX, selective and non-selective non-steroidal anti-inflammatory drugs. Ann Rheum Dis. 2003;62(6):501-509. doi:10.1136/ard.62.6.501

4. Kaur G, Silakari O. Multiple target-centric strategy to tame inflammation. Future Med Chem. 2017;9(12):1361-1376. doi:10.4155/fmc2017-0050
5. Schaible AM, Traber H, Temml V, et al. Potent inhibition of human 5-lipoxygenase and microsomal prostaglandin E2 synthase-1 by the anti-carcinogenic and anti-inflammatory agent embelin. Biochem Pharmacol. 2001;21(1):1-15. doi:10.1016/j.bcp.2013.04.015

6. Koeberle A, Rossi A, Zettl H, et al. The molecular pharmacology and in vivo activity of 2-(4-chloro-6-(2,3-dimethylphenylamino)pyrimidin-2-ylthio)octanoic acid (YS121), a dual inhibitor of microsomal prostaglandin E2 synthase-1 and 5-lipoxygenase. J Pharmacol Exp Ther. 2010;332(3):840-848.

7. Bruno F, Errico S, Pace S, et al. Structural insight into the optimization of ethyl 5-hydroxybenzo[g]indol-3-carboxylates and their bioisosteric analogues as 5-LO/m-PGES-1 dual inhibitors able to suppress inflammation. Eur J Med Chem. 2018;155:946-960.

8. Liedtke AJ, Keck PR, Lehmann F, Koeberle A, Werz O, Laufer SA. Arylpyrrolizines as inhibitors of microsomal prostaglandin E2 synthase-1 (mPGES-1) or as dual inhibitors of mPGES-1 and 5lipoxygenase (5-LOX). J Med Chem. 2009;52(15):4968-4972.

9. Koeberle A, Siemoneit U, Bühring U, et al. Licofelone suppresses prostaglandin E2 formation by interference with the inducible microsomal prostaglandin E2 synthase-1. J Pharmacol Exp Ther. 2008;326 (3):975-982.

10. Mohammed A, Janakiram NB, Li Q, et al. Chemoprevention of colon and small intestinal tumorigenesis in APCMin/+ mice by licofelone, a novel dual 5-LOX/COX inhibitor: potential implications for human colon cancer prevention. Cancer Prev Res. 2011;4(12):2015-2026. doi:10.1158/1940-6207.CAPR-11-0233

11. Madka V, Mohammed A, Li Q, et al. Chemoprevention of urothelial cell carcinoma growth and invasion by the dual COX-LOX inhibitor licofelone in UPII-SV40T transgenic mice. Cancer Prevent Res. 2014;7(7):708-716. doi:10.1158/1940-6207.CAPR-14-0087

12. Kumar G, Patlolla JM, Madka V, et al. Simultaneous targeting of 5LOX-COX and ODC block NNK-induced lung adenoma progression to adenocarcinoma in A/J mice. Am J Cancer Res. 2016;6(5):894909.

13. Rao CV, Janakiram NB, Madka V, et al. Simultaneous targeting of 5LOX-COX and EGFR blocks progression of pancreatic ductal adenocarcinoma. Oncotarget. 2015;6(32):33290-33305. doi:10.18632/ oncotarget.5396

14. Raynauld J-P, Martel-Pelletier J, Bias P, et al. Protective effects of licofelone, a 5-lipoxygenase and cyclo-oxygenase inhibitor, versus naproxen on cartilage loss in knee osteoarthritis: a first multicentre clinical trial using quantitative MRI. Ann Rheum Dis. 2009;68 (6):938-947. doi:10.1136/ard.2008.088732

15. Aidin ST, Mohammad A, Aidin E. Effects of licofelone, a novel 5LOX inhibitor, in comparison to celecoxib on gastric mucosa of dogs. Veterinary. Sci Dev. 2017;7(6245):69-71.

16. Erlanson DA, Davis BJ, Jahnke W. Fragment-Based Drug Discovery: advancing Fragments in the Absence of Crystal Structures. Cell Chem Biol. 2019;26(1):9-15. doi:10.1016/j.chembiol.2018.10.001

17. Sjögren T, Nord J, Ek M, Johansson P, Liu G, Geschwindner S. Crystal structure of microsomal prostaglandin E2 synthase provides insight into diversity in the MAPEG superfamily. Proc Natl Acad Sci U S A. 2013;110(10):3806-3811. doi:10.1073/pnas.1218504110

18. Ding K, Zhou Z, Hou S. Zhou, Z., Hou, S. et al. Structure-based discovery of mPGES-1 inhibitors suitable for preclinical testing in wild-type mice as a new generation of anti-inflammatory drugs. Sci Rep. 2018;8(1):5205. doi:10.1038/s41598-018-23482-4

19. Terzić J, Grivennikov S, Karin E, Karin M. Inflammation and colon cancer. Gastroenterol. 2010;138(6):2101-2114. doi:10.1053/j. gastro.2010.01.058

20. Janakiram NB, Rao CV. The role of inflammation in colon cancer. Adv Exp Med Biol. 2014;816:25-52.

21. Kim W-I, Choi K-A, Do H-S, Yu Y-G. Expression and purification of human mPGES-1 in E. coli and identification of inhibitory compounds from a drug-library. BMB Rep. 2008;41(11):808-813. doi:10.5483/BMBRep.2008.41.11.808 
22. Lazarus M, Eguchi N, Matsumoto S, et al. Species-specific expression of microsomal prostaglandin E synthase-1 and cyclooxygenases in male monkey reproductive organs. Prostaglandins Leukotrienes Essential Fatty Acids. 2004;71(4):233-240. doi:10.1016/j. plefa.2004.03.018

23. Lazarus M, Munday CJ, Eguchi N, et al. Immunohistochemical localization of microsomal PGE synthase-1 and cyclooxygenases in male mouse reproductive organs. Endocrinology. 2002;143(6):2410 2419. doi:10.1210/endo.143.6.8872

24. Neeraja S, Sreenath AS, Reddy PR, Reddanna P. Expression of cyclooxygenase-2 in rat testis. Reproduct BioMed Online. 2003;6 (3):302-309. doi:10.1016/S1472-6483(10)61849-4

25. Rao CV, Janakiram NB, Gali H, Mohammed A, Pathuri G (2019). Licofelone derivatives and methods of use. U.S. Patent No. US10206904B2, issued: 02/19/2019.

26. Starr T, Bauler TJ, Malik-Kale P, Steele-Mortimer O, Ko DC. The phorbol 12-myristate-13-acetate differentiation protocol is critical to the interaction of THP-1 macrophages with Salmonella Typhimurium. PLoS One. 2018;13(3):e0193601. doi:10.1371/journal.pone.0193601

27. Janakiram NB, Mohammed A, Bryant $\mathrm{T}$, et al. Improved innate immune responses by Frondanol A5, a sea cucumber extract, prevent intestinal tumorigenesis. Cancer Prevent Res. 2015;8(4):327-337. doi:10.1158/1940-6207.CAPR-14-0380

28. Yang J-M, Chen C. C. GEMDOCK: a generic evolutionary method for molecular docking. Proteins. 2004;55(2):288-304. doi:10.1002/ prot.20035

29. Yarla NS, Azad R, Basha M, et al. 5-Lipoxygenase and cyclooxygenase inhibitory dammarane triterpenoid 1 from Borassus flabellifer seed coat inhibits tumor necrosis factor- $\alpha$ secretion in LPS-induced THP-1 human monocytes and induces apoptosis in MIA PaCa-2 pancreatic cancer cells. Anti Cancer Agents in Med. 2015;15 (8):1066-1077. doi:10.2174/1871520615666150202110936

30. Liu F, Chen X, Allali-Hassani A, et al. Discovery of a 2,4-diamino-7aminoalkoxyquinazoline as a potent and selective inhibitor of histone lysine methyltransferase G9a. J Med Chem. 2009;52(24):7950-7953. doi:10.1021/jm901543m

31. Wiedemann C, Bellstedt P, Görlach GM. CAPITO - a web serverbased analysis and plotting tool for circular dichroism data. Bioinformatics. 2013;29(14):1750-1757. doi:10.1093/bioinformatics/btt278

32. Naret T, Khelifi I, Provot O, et al. 1,1-Diheterocyclic ethylenes derived from quinaldine and carbazole as new tubulin-polymerization inhibitors: synthesis, metabolism, and biological evaluation. $\mathrm{J} \mathrm{Med}$ Chem. 2019;62(4):1902-1916. doi:10.1021/acs.jmedchem.8b01386

33. Veber DF, Johnson SR, Cheng H-Y, Smith BR, Ward KW, Kopple KD. Molecular properties that influence the oral bioavailability of drug candidates. J Med Chem. 2002;45(12):2615-2623. doi:10.1021/ jm020017n

34. Gupta A, Chaudhary N, Kakularam KR, Pallu R, Polamarasetty A, Zheng J. The augmenting effects of desolvation and conformational energy terms on the predictions of docking programs against mPGES-1. PLoS One. 2015;10(8):e0134472. doi:10.1371/journal. pone. 0134472

35. Richardson SJ, Bai A, Kulkarni AA, Moghaddam MF. Efficiency in drug discovery: liver S9 fraction assay as a screen for metabolic stability. Drug Metabolism Lett. 2016;10(2):83-90. doi:10.2174/ 1872312810666160223121836

36. Chandrakesan P, Roy B, Jakkula LU, et al. Utility of a bacterial infection model to study epithelial-mesenchymal transition, mesenchymal-epithelial transition or tumorigenesis. Oncogene. 2014;33(20):2639-2654. doi:10.1038/onc.2013.210

37. Rayavarapu S, Yarla NS, Kadiri SK, et al. Synthesis of Saccharumoside-B analogue with potential of antiproliferative and pro-apoptotic activities. Sci Rep. 2017;7(1):8309.
38. Di Lorenzo A, Fernández-Hernando C, Cirino G, Sessa WC. Akt1 is critical for acute inflammation and histamine-mediated vascular leakage. Proc Nat Acad Sci. 2009;106(34):14552-14557. doi:10.1073/ pnas.0904073106

39. National Research Council (US). Committee for the Update of the Guide for the Care and Use of Laboratory Animals. Guide for the Care and Use of Laboratory Animals. 8th edition ed. Washington (DC): National Academies Press (US); 2011. Available from: https:/www.ncbi.nlm.nih. gov/books/NBK54050/. Accessed December 7, 2020.

40. Chattopadhyay M, Kodela R, Duvalsaint PL, Kashfi K. Gastrointestinal safety, chemotherapeutic potential, and classic pharmacological profile of NOSH-naproxen (AVT-219) a NO and H2 S-releasing hybrid. Pharmacol Res Perspect. 2016;4(2):e00224. doi:10.1002/prp2.224

41. Liang J, Huang -Y-Y, Zhou Q, et al. Discovery and optimization of $\alpha$ mangostin derivatives as novel PDE4 inhibitors for the treatment of vascular dementia. J Med Chem. 2020;63(6):3370-3380. doi:10.1021/acs.jmedchem.0c00060

42. Baell JB, Holloway GA. New substructure filters for removal of pan assay interference compounds (PAINS) from screening libraries and for their exclusion in bioassays. J Med Chem. 2010;53(7):2719-2740. doi:10.1021/jm901137j

43. He S, Wu Y, Yu D, Lai L. Microsomal prostaglandin E synthase-1 exhibits one-third-of-the-sites reactivity. Biochem J. 2011;440(1):1321. doi:10.1042/BJ20110977

44. Hamberg M, Wetterholm A, Hansson H, Samuelsson B, Haeggström JZ. Mutation of a critical arginine in microsomal prostaglandin e synthase-1 shifts the isomerase activity to a reductase activity that converts prostaglandin h2 into prostaglandin F. J Biol Chem. 2009;284(1):301-305. doi:10.1074/jbc.M808365200

45. Raouf J, Rafique N, Goodman MC, et al. Arg126 and Asp49 are essential for the catalytic function of microsomal prostaglandin $\mathrm{e}_{2}$ synthase 1 and ser127 is not. PLoS One. 2016;11(9):e0163600. doi:10.1371/journal.pone.0163600

46. Gupta A, Aparoy P. Insights into the structure activity relationship of mPGES-1 inhibitors: hints for better inhibitor design. Int $\mathrm{J}$ Biol Macromolecules. 2016;88:624-632. doi:10.1016/j.ijbiomac.2016.03.038

47. Gilbert NC, Bartlett SG, Waight MT, et al. The structure of human 5lipoxygenase. Science. 2011;331(6014):217-219. doi:10.1126/ science. 1197203

48. Rowlinson SW, Kiefer JR, Prusakiewicz JJ, et al. A novel mechanism of cyclooxygenase-2 inhibition involving interactions with Ser-530 and Tyr-385. J Biol Chem. 2011;4(12):2015-2026. doi:10.1074/jbc. M305481200

49. Chandrasekharan NV, Simmons DL. The cyclooxygenases. Genome Biol. 2019;26(1):241. doi:10.1186/gb-2004-5-9-241

50. Bogdan D, Falcone J, Kanjiya MP, et al. Fatty acid-binding protein 5 controls microsomal prostaglandin E synthase 1 (mPGES-1) induction during inflammation. J Biol Chem. 2018;293(14):5295-5306.

51. Wallace JL, McKnight W, Reuter BK, Vergnolle N. NSAID-induced gastric damage in rats: requirement for inhibition of both cyclooxygenase 1 and 2. Gastroenterol. 2000;119(3):706-714.

52. Chattopadhyay M, Velazquez CA, Pruski A, et al. Comparison between 3-Nitrooxyphenyl acetylsalicylate (NO-ASA) and O2-(acetylsalicyloxymethyl)-1-(pyrrolidin-1-yl)diazen-1-ium-1,2-diolate (NONO-ASA) as safe anti-inflammatory, analgesic, antipyretic, antioxidant prodrugs. J Pharmacol Exp Ther. 2010;335(2):443-450.

53. Wang D, Fu L, Sun H, Guo L, dubois RN. Prostaglandin $\mathrm{E}_{2}$ promotes colorectal cancer stem cell expansion and metastasis in mice. Gastroenterol. 2015;149(7):1884-1895.e4.

54. Ana SN, Andreia SB, Elisabete CC, André FM, Ilídio JC. 3D tumor spheroids as in vitro models to mimic in vivo human solid tumors resistance to therapeutic drugs. Biotechnol Bioeng. 2019;116(1):206226. 


\section{Publish your work in this journal}

The Journal of Inflammation Research is an international, peerreviewed open-access journal that welcomes laboratory and clinical findings on the molecular basis, cell biology and pharmacology of inflammation including original research, reviews, symposium reports, hypothesis formation and commentaries on: acute/chronic inflammation; mediators of inflammation; cellular processes; molecular mechanisms; pharmacology and novel anti-inflammatory drugs; clinical conditions involving inflammation. The manuscript management system is completely online and includes a very quick and fair peerreview system. Visit http://www.dovepress.com/testimonials.php to read real quotes from published authors. 